\title{
Tissue-specific and interferon-inducible expression of nonfunctional ACE2 through endogenous retroelement co-option
}

\author{
Kevin W. Ng1, Jan Attig ${ }^{1}$, William Bolland', George R. Young ${ }^{\circledR 2}$, Jack Major ${ }^{3}$, Antoni G. Wrobel ${ }^{4}$, \\ Steve Gamblin ${ }^{4}{ }^{4}$, Andreas Wack ${ }^{3}$ and George Kassiotis ${ }^{1,5} \bowtie$
}

\begin{abstract}
Angiotensin-converting enzyme 2 (ACE2) is an entry receptor for severe acute respiratory syndrome coronavirus 2 (SARS-CoV-2) and a regulator of several physiological processes. ACE2 has recently been proposed to be interferon (IFN) inducible, suggesting that SARS-CoV-2 may exploit this phenomenon to enhance viral spread and questioning the efficacy of IFN treatment in coronavirus disease 2019. Using a recent de novo transcript assembly that captured previously unannotated transcripts, we describe a new isoform of ACE2, generated by co-option of intronic retroelements as promoter and alternative exon. The new transcript, termed MIRb-ACE2, exhibits specific expression patterns across the aerodigestive and gastrointestinal tracts and is highly responsive to IFN stimulation. In contrast, canonical ACE2 expression is unresponsive to IFN stimulation. Moreover, the MIRb-ACE2 translation product is a truncated, unstable ACE2 form, lacking domains required for SARS-CoV-2 binding and is therefore unlikely to contribute to or enhance viral infection.
\end{abstract}

FNs represent the first line of defense against viruses in humans and other jawed vertebrates ${ }^{1}$. Recognition of viral products in an infected cell results in autocrine and paracrine signaling to induce an antiviral state characterized by expression of a module of IFN-stimulated genes (ISGs) that restrict viral replication and spread ${ }^{1,2}$. Indeed, recombinant IFN is often given as first-line therapy in viral infection ${ }^{3}$, and preliminary results suggest that IFN treatment may be effective against coronavirus disease 2019 (COVID-19) $)^{4,5}$.

IFN signaling results in rapid upregulation of several hundred ISGs, including genes that inhibit various stages of viral entry and replication, as well as transcription factors that further potentiate the IFN response ${ }^{1,2}$. Given that unchecked IFN signaling and inflammation can result in immunopathology, ISGs are subject to complex regulatory mechanisms ${ }^{6}$.

At the transcriptional level, long terminal repeats (LTRs), derived from endogenous retroviruses and other LTR retroelements, as well as regulatory sequences in non-LTR retroelements, serve as cis-regulatory enhancers for a number of ISGs and are required for their induction ${ }^{7}$. Adding to this regulatory complexity, many retroelements are themselves IFN-responsive promoters and are upregulated following viral infection or in IFN-driven autoimmunity ${ }^{8-11}$.

The coevolution of viruses and hosts has resulted in a number of strategies by which viruses evade or subvert IFN responses ${ }^{12}$. Compared with other respiratory viruses, SARS-CoV-2 elicits a weak IFN response despite strong induction of other chemokines ${ }^{13}$. Although the mechanism by which SARS-CoV-2 dampens IFN responses remains unclear, the ORF3b, ORF6 and nucleoprotein of the closely related SARS-CoV function as IFN antagonists ${ }^{14}$. SARS-CoV-2 uses ACE2 as its primary receptor ${ }^{15,16}$, and recent work suggested that SARS-CoV-2 may hijack the IFN response by inducing ACE2 expression ${ }^{17}$. By integrating multiple human, macaque and mouse single-cell RNA-sequencing (scRNA-seq) datasets, Ziegler et al. identified $A C E 2$ as a primate-specific ISG upregulated following viral infection or IFN treatment ${ }^{17}$. Use of an ISG as a viral receptor would result in a self-amplifying loop to increase local viral spread and calls into question the efficacy and safety of recombinant IFN treatment in patients with COVID-19.

Using our recent de novo transcriptome assembly ${ }^{18}$, we identify a new, truncated $A C E 2$ transcript, termed $M I R b$-ACE2, initiated at intronic $M I R b$ and $L T R 16 A 1$ retroelements that serve as a cryptic promoter, alternative exon and splice site. Notably, we find that the truncated $M I R b-A C E 2$ and not full-length ACE2 is the IFN-inducible isoform and is strongly upregulated in viral infection and following IFN treatment. Importantly, the protein product of the $M I R b$-ACE2 transcript does not contain the amino acid residues required for SARS-CoV-2 attachment and entry and is additionally posttranslationally unstable. These findings have important implications for the understanding of ACE2 expression and regulation and thus for SARS-CoV-2 tropism and treatment.

\section{Results}

$M I R b-A C E 2$ is a tissue-specific new isoform of $A C E 2$. Our recent de novo cancer transcriptome assembly ${ }^{18}$ identified a chimeric transcript formed by splicing between annotated exons of ACE2 and an LTR16A1 retroelement, integrated in intron 9 of the ACE2 locus. This transcript, which we refer to here as MIRb-ACE2, includes exons 10-19 of ACE2 (Fig. 1a). Splicing between the LTR16A1 retroelement and exon 10 of $A C E 2$ was highly supported by splice junction analysis of RNA-seq data from The Cancer Genome Atlas (TCGA) lung adenocarcinoma (LUAD) and lung squamous cell carcinoma (LUSC) cohorts (Fig. 1a). To identify potential transcription start site(s) of the MIRb-ACE2 transcript, we inspected promoter-based expression analyses of the FANTOM5 dataset,

'Retroviral Immunology, The Francis Crick Institute, London, UK. ${ }^{2}$ Retrovirus-Host Interactions, The Francis Crick Institute, London, UK. ${ }^{3}$ mmunoregulation, The Francis Crick Institute, London, UK. ${ }^{4}$ Structural Biology of Disease Processes, The Francis Crick Institute, London, UK. ${ }^{5}$ Department of Medicine, Faculty of Medicine, Imperial College London, London, UK.凶e-mail: george.kassiotis@crick.ac.uk 
a

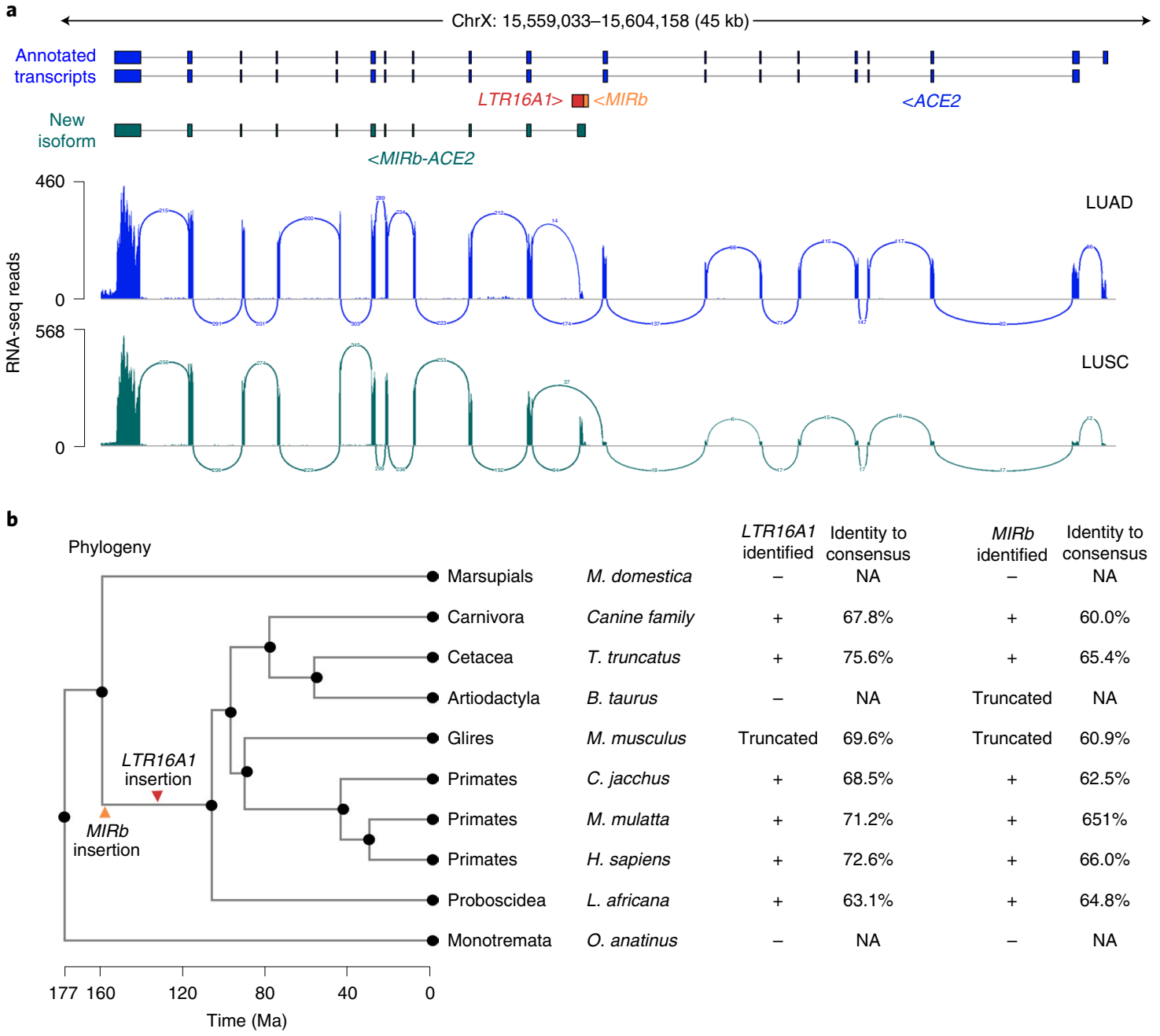

C Alignment

LTR16A1...length (nucleotides)

MIRb...length (nucleotides)

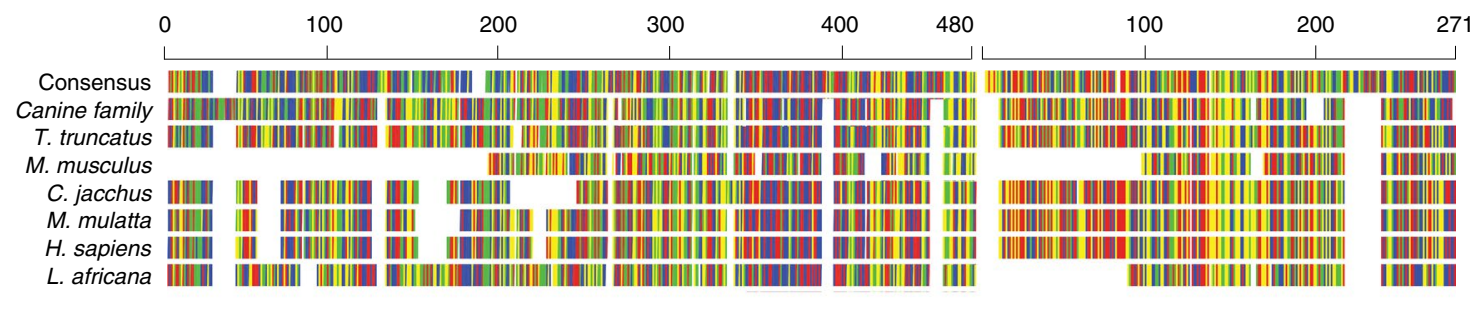

$\square \mathrm{T} \quad \mathrm{A} \square \mathrm{C}$

Fig. 1 Identification of the new MIRb-ACE2 isoform. a, GENCODE annotated transcripts at the ACE2 locus, intronic position of the MIRb and LTR16A1 elements, structure of the new MIRb-ACE2 isoform and RNA-seq traces of composite LUAD and LUSC samples. Also shown is splice junction analysis of the same RNA-seq samples. b. Phylogenetic analysis of the MIRb and LTR16A1 sequences in the indicated representative mammalian species and percentage sequence identity to the consensus MIRb and LTR16A1 sequences. The arrows indicate the estimated timing of ancestral integrations of the $M I R b$ and LTR16A1 elements, respectively. Ma, million years ago; NA, not applicable. c, Alignment of the MIRb and LTR16A1 sequences in the indicated representative mammalian species and of the consensus MIRb and LTR16A1 sequences.

which indicated peaks in the LTR16A1 retroelement and the immediately upstream $M I R b$ retroelement in the same intronic region (Extended Data Fig. 1). FANTOM5 CAGE peak distribution over the LTR16A1 and MIRb retroelements exhibited cell-type specificity to a certain degree, with peaks residing almost exclusively in $M I R b$ in bronchial epithelial cells but extending to LTR16A1 in HEK293 cells (Extended Data Fig. 1). Both LTR16A1 and MIRb retroelements contained multiple transcription factor binding sites, with IRF-1 and IRF-2 binding sites and TATA-box residing in $M I R b$
(Extended Data Fig. 2). To further define the transcription start site(s), we performed $5^{\prime}$ rapid amplification of cDNA ends (RACE) PCR, followed by deep sequencing of the PCR products, amplified from normal human bronchial epithelial (NHBE) cells or human squamous cell carcinoma (SCC) cell lines SCC-4 and SCC-25, treated with recombinant IFN- $\alpha$ (Extended Data Fig. 2). Consistent with FANTOM5 CAGE data, 5' RACE analysis showed multiple peaks in both LTR16A1 and MIRb, again with evidence of celltype specificity in their relative utilization (Extended Data Fig. 2). 
These results suggested that the $M I R b$ and $L T R 16 A 1$ retroelements acted as a cryptic promoter for the $M I R b-A C E 2$ transcript, with transcription start sites distributed across these two retroelements.

Phylogenetic analysis of the respective LTR16A1 and MIRb elements in the ACE2 loci of representative mammalian species indicated that the ancestral integrations predated estimated dates of mammalian radial divergence (Fig. 1b). Indeed, comparative genomic analysis produced good alignment of the LTR16A1 and $M I R b$ integrations across a variety of species, with humans, dogs, and dolphins showing above $60 \%$ sequence identity to the mammalian consensus sequences of LTR16A1 and MIRb (Fig. 1b,c). Of note, the LTR16A1 and MIRb integrations were also present but truncated in the murine ACE2 locus (Fig. 1b,c), further supported by $\mathrm{LASTz}$ human-mouse whole-genome alignment (Ensembl Compara databases). To further support the intronic initiation of the chimeric $M I R b$-ACE2 transcript in species other than humans, we performed quantitative PCR with reverse transcription (RT-qPCR) analyses using primers complementary to the respective LTR16A1 and ACE2 exon 10 sequences in the African green monkey, dog, rabbit or mouse genome (Extended Data Fig. 3). Whereas all cell lines from these representative species expressed the canonical $A C E 2$ transcript, the $M I R b-A C E 2$ transcript was detected in $C V-1$ but not Vero cells (both from African green monkey; Extended Data Fig. 3). The $M I R b-A C E 2$ transcript was undetectable in leporine R9ab and murine MCA-38 cells but was present in canine MDCK cells (Extended Data Fig. 3), consistent with the high degree of LTR16A1 and MIRb element conservation in the latter species family. Therefore, $M I R b-A C E 2$ expression in humans likely represents a common mammalian feature that has been lost in some, but not all, other mammalian species.

To assess the relative expression of ACE2 and MIRb-ACE2 isoforms, we quantified expression of both transcripts across tissue types in the TCGA and Genotype-Tissue Expression (GTEx) cohorts. Consistent with recent reports ${ }^{17,19}$, full-length $A C E 2$ was expressed predominantly in the healthy intestine and kidney and tumors of the same histotypes (Extended Data Fig. 4). Expression of $M I R b$-ACE2 followed a similar overall pattern but with notable expression also in healthy testis, likely owing to retroelement activation as part of epigenetic reprogramming during spermatogenesis.

However, despite similar histotype distribution of ACE2 and $M I R b$-ACE2 expression, the ratio of the two isoforms was characteristically different between distinct histotypes and tumor types. For example, in larger TCGA patient cohorts, LUAD samples expressed higher levels of ACE2 than of MIRb-ACE2 (mean ACE2/ $M I R b$-ACE2 ratio=5.63), whereas LUSC samples showed the opposite phenotype with higher expression of MIRb-ACE2 (mean $A C E 2 / M I R b-A C E 2$ ratio=0.87; Fig. 2a,b). ACE2 and MIRb-ACE2 expression and their ratios were not affected by patient sex, arguing against a strong effect of the $\mathrm{X}$-chromosomal location of ACE2 on either isoform expression (Fig. 2a,b). ACE2 and MIRb-ACE2 exhibited characteristic expression also within tumor types with only weak correlation between the two in the same tumor type $\left(R^{2}=0.252\right.$ for LUAD; $R^{2}=0.337$ for LUSC), suggesting partly independent regulation.

In healthy lung, expression of $A C E 2$ and $M I R b-A C E 2$ was similar to that in LUAD, with the balance slightly in favor of the full-length isoform (mean $A C E 2 / M I R b-A C E 2$ ratio = 2.73; Fig. $2 \mathrm{c}$ ). In contrast, healthy colon expressed considerably higher levels of ACE2 and $M I R b-A C E 2$, specifically of the full-length isoform (mean ACE2/ $M I R b-A C E 2$ ratio=26.37; Fig. 2d). These differences in ACE2 and $M I R b-A C E 2$ expression between healthy lung and colon were again independent of sex (Fig. 2c,d).

Tissue-specific patterns of ACE2 and MIRb-ACE2 expression suggested dependency on cell lineage or identity. Alternatively, they could reflect transient adaptations to the local microenvironment, such as oxygen or microbiota composition differences between lung and intestine, or even differences in cellular composition between the different compartments. To examine whether patterns of ACE2 and $M I R b-A C E 2$ expression are linked to cell identity, we examined RNA-seq data from 933 cancer cell lines from The Cancer Cell Line Encyclopedia (CCLE). These data represent homogeneous cell populations, grown under standardized conditions, independently of environmental influences. Again, expression of ACE2 and $M I R b$-ACE2 was characteristically different between different cell lines and correlated with their anatomical origin (Fig. 3a-d). Cell lines with the highest expression of $M I R b$-ACE2 were derived from the upper aerodigestive tract, including the mouth and nose (mean $A C E 2 / M I R b-A C E 2$ ratio $=0.72)$, followed by esophageal cell lines (mean $A C E 2 / M I R b-A C E 2$ ratio $=1.66$ ) and lung cell lines (mean $A C E 2 / M I R b-A C E 2$ ratio $=6.27)$. Consistent with data from primary biopsies, cells lines from the large intestine exhibited the highest expression of $A C E 2$, with minimal expression of $M I R b-A C E 2$ (mean $A C E 2 / M I R b-A C E 2$ ratio=16.97). The low $A C E 2 / M I R b-A C E 2$ ratio in the upper aerodigestive tract was significant when compared with other locations, including the lung and large intestine $(P=0.0035$ and $P=0.0023$, respectively; Student's $t$-test).

Together, these results uncover the transcription of a new ACE2 isoform, initiated at the intronic MIRb-LTR16A1 retroelements, in a characteristic pattern of expression, forming a gradient from the upper aerodigestive tract (highest $M I R b-A C E 2$ expression) to the large intestine (highest $A C E 2$ expression).

$M I R b-A C E 2$ and not $A C E 2$ transcription is IFN responsive. ACE2 has recently been described as a human ISG, upregulated at the mRNA level following viral infection or IFN treatment ${ }^{17,20}$. However, this conclusion was based mostly on analysis of scRNA-seq data that might not have sufficient resolution to distinguish the two isoforms. Indeed, inspection of public scRNA-seq data (GSE134355) ${ }^{21}$, demonstrated the limitation of such technologies, with RNA-seq reads mapping exclusively to the shared $3^{\prime}$ terminal exon of the ACE2 transcripts, and therefore unable to discriminate between the isoforms (Extended Data Fig. 5).

To investigate the inducibility of the two isoforms by IFN or viral infection, we reanalyzed public RNA-seq data (GSE147507) from NHBE cells, treated with recombinant IFN- $\beta$ or infected with SARS-CoV-2, influenza A virus (IAV) or IAV lacking the viral NS1 protein (IAV $\Delta \mathrm{NS} 1)^{13}$. None of the treatments increased expression of full-length ACE2 (Fig. 4a). In stark contrast, MIRb-ACE2 expression was strongly elevated by both IAV $\Delta$ NS1 infection and recombinant IFN- $\beta$ treatment, compared with mock treatment ( $P=0.0005$ and $P=0.0054$, respectively; Student's $t$-test). Similar results were also obtained with analysis of lung cancer Calu- 3 cells. In the absence of stimulation, Calu-3 cells expressed exclusively the full-length ACE2 isoform (Fig. 4b). SARS-CoV-2 infection did not affect levels of $A C E 2$ expression but noticeably induced MIRb-ACE2 expression (Fig. 4b). Lastly, analysis of RNA-seq data from explanted lung tissue from a single patient with COVID-19 demonstrated elevated expression of $M I R b-A C E 2$ but not of $A C E 2$, compared with healthy lung tissue (Fig. 4c); albeit statistical comparisons were not possible in this case.

To further confirm the IFN-responsiveness exclusively of $M I R b$-ACE2 expression, we used SCC- 4 and SCC- 25 cells, which express both isoforms. Compared with mock treatment, addition of recombinant IFN- $\alpha$ or IFN- $\gamma$ had a minimal effect on ACE2 expression in SCC- 4 cells and no effect in SCC- 25 cells (Fig. 4d). This contrasted with very strong induction ( 15 fold) of MIRb-ACE2 expression by either type of IFN in both cell lines (Fig. 4d). Lack of $A C E$ responsiveness to IFN stimulation was additionally confirmed at the protein level, where neither IFN- $\alpha$ nor IFN- $\gamma$ affected levels of full-length ACE2, detected by western blotting in SCC-4 and SCC-25 cells or in A549 cells, which express neither isoform and were used as a negative control (Fig. 4e). Splicing from the 


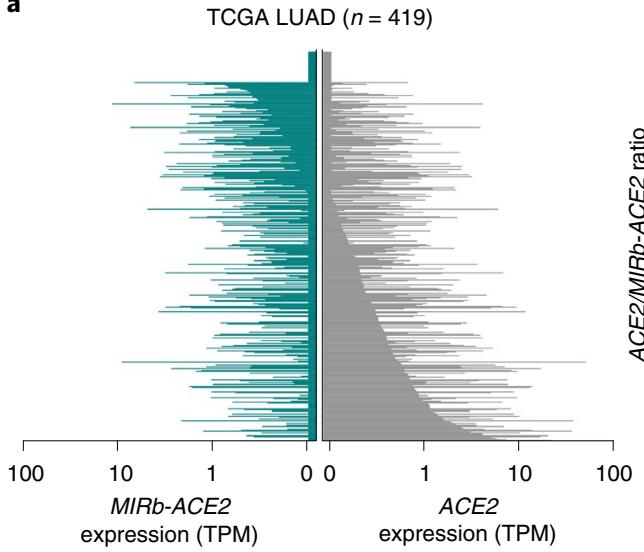

$c$

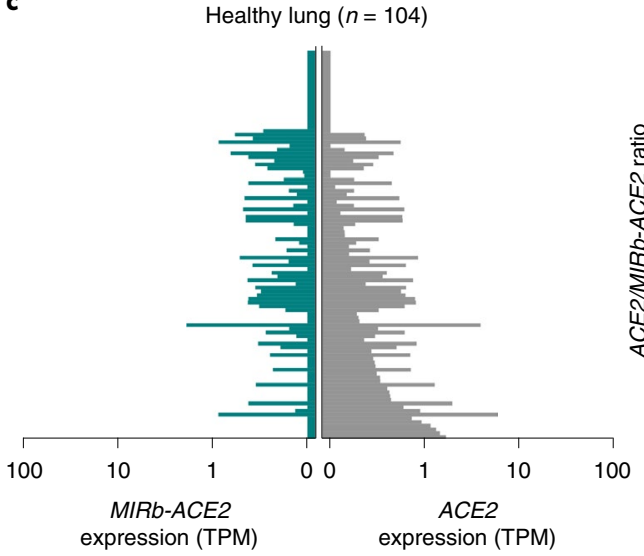

b

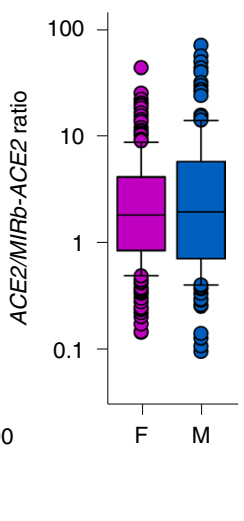

TCGA LUSC $(n=362)$

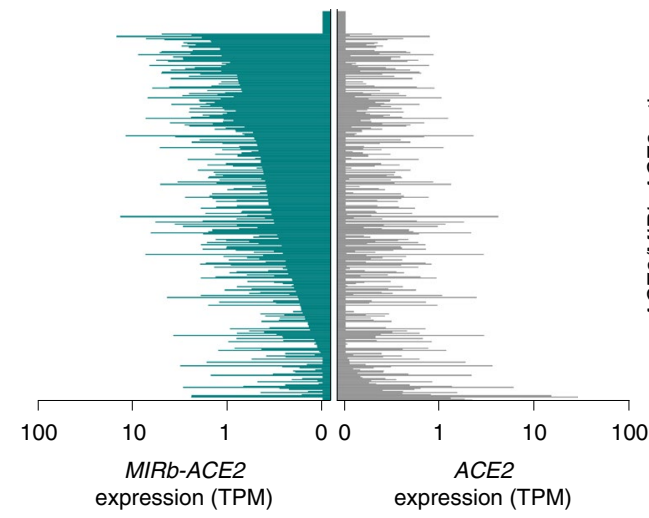

d
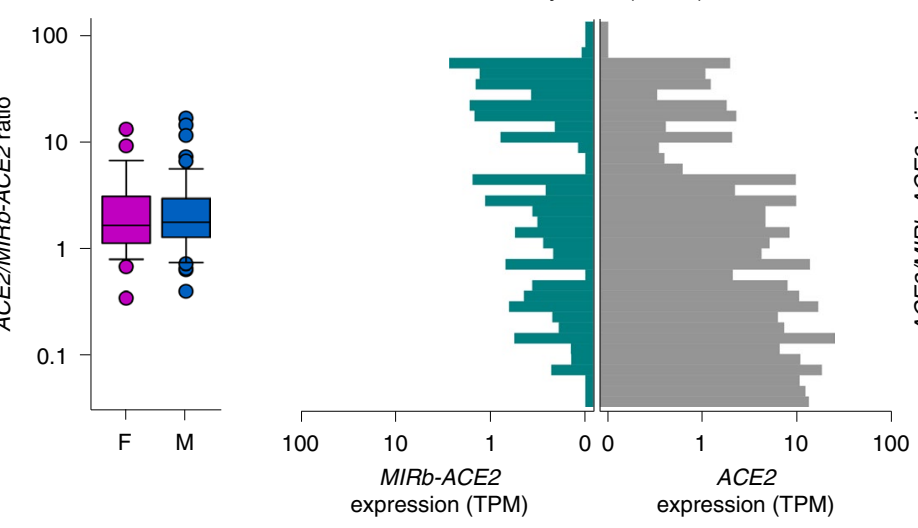
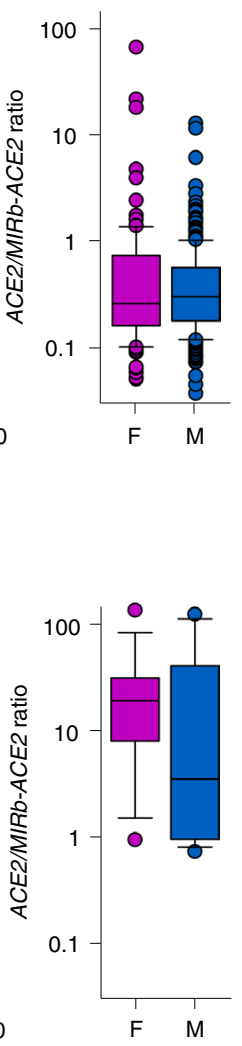

Fig. 2 | ACE2 and MIRb-ACE2 isoform expression in cancer and healthy tissues. a, ACE2 and MIRb-ACE2 isoform expression in LUAD samples (left) and ratio of the two isoforms in female $(F ; n=225)$ and male $(M ; n=161)$ samples (right). $\mathbf{b}, A C E 2$ and MIRb-ACE2 isoform expression in LUSC samples and ratio of the two isoforms ( $F, n=100 ; M, n=241)$. c, ACE2 and MIRb-ACE2 isoform expression in healthy lung samples and ratio of the two isoforms ( $F$, $n=29 ; M, n=54)$. d, ACE2 and MIRb-ACE2 isoform expression in healthy colon samples and ratio of the two isoforms ( $F$, $n=18 ; M, n=16)$. In a-d, each bar represents an individual sample. Box plots show the upper and lower quartiles, center lines show the median, whiskers represent $1.5 \times$ the interquartile range and individual points represent outliers. TPM, transcripts per million.

LTR16A1 retroelement to exon 10 of ACE2 is in frame and, therefore, the last 449 amino acids of ACE2 are also present in the putative MIRb-ACE2 protein. Of note, despite strong upregulation at the mRNA level and despite using polyclonal antibodies (ab15348) targeting the $\mathrm{C}$ terminus of ACE2 present in both protein products, we were unable to detect a truncated form that would correspond to the MIRb-ACE2 translation product in SCC- 4 or SCC- 25 cells (Fig. 4e).

To confirm the differential IFN inducibility of $A C E 2$ and $M I R b-A C E 2$ expression, we stimulated NHBE cells with IFN- $\alpha$, IFN- $\beta$ or IFN- $\lambda$, as previously described ${ }^{22}$. Again, treatment with none of the IFNs had any measurable effect on ACE2 expression in these primary cells (Fig. 4f). This contrasted with robust induction of MIR $b$-ACE2 expression, particularly by IFN- $\alpha$ (Fig. 4f).

Collectively, these data demonstrate that type I, II and III IFNs stimulate transcription of the ACE2 isoform driven by the alternative MIRb-LTR16A1, but not the canonical ACE2 promoter.

The MIRb-ACE2 protein product is not stable. The MIRb-ACE2 isoform is predicted to encode a truncated ACE2 product (amino acids 357-805), and exonization of the LTR16A1 element creates a new ten amino acid N-terminal sequence (MREAGWDKGG) in the putative translation product (Extended Data Fig. 6). Importantly, this predicted protein lacks the first 356 amino acids, including the signal peptide, substrate-binding site and domains that interact with SARS-CoV and SARS-CoV-2 spike glycoproteins (Extended Data Fig. 6). Despite sharing the C-terminal half of full-length ACE2, which was readily detectable, endogenously produced MIRb-ACE2 protein was not detectable in SCC-4 and SCC-25 cells naturally expressing the $M I R b-A C E 2$ transcript, by western blotting using polyclonal anti-ACE2 antibodies (ab15348; Fig. 4e). To explore the protein-coding potential of the MIRb-ACE2 transcript, we cloned the coding sequences of both isoforms into the pcDNA3.1 mammalian expression vector and transfected HEK293T cells, which do not endogenously express ACE2, thus preventing confounding of the detection of ACE2 produced following transfection ${ }^{16,23}$. While ACE2-transfected HEK293T cells produced detectable full-length ACE2, no protein of the predicted size was detectable in $M I R b$-ACE2-transfected cells (Extended Data Fig. 7 ), in agreement with results using SCC-4 and SCC-25 cells (Fig. 4e). In independently reported findings ${ }^{24}$, endogenously produced MIRb-ACE2 protein could not be detected by western blotting using the same polyclonal anti-ACE2 serum (ab15348). However, a Myc-DDK-tagged or green fluorescent protein (GFP)-tagged MIRb-ACE2 protein product was detected following overexpression in T24 cells in the same study ${ }^{24}$. Moreover, a separate study ${ }^{25}$ reported detection of the putative MIRb-ACE2 protein in primary nasal epithelial cells by western blotting using the same polyclonal anti-ACE2 serum (ab15348), raising the possibility that the protein can indeed be translated. 
a

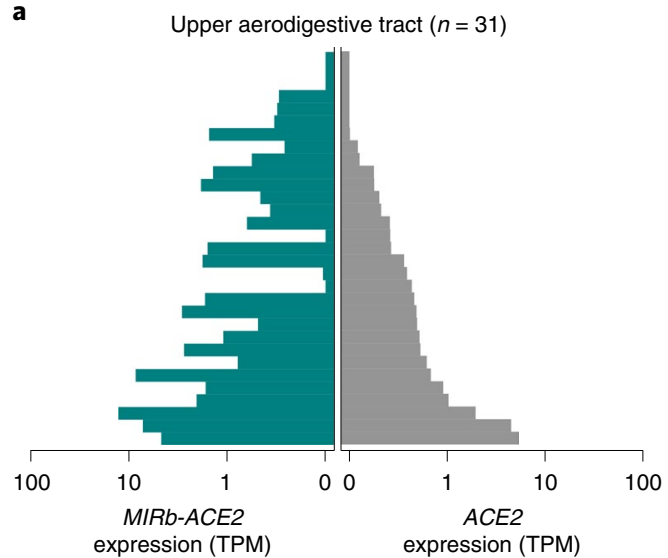

C

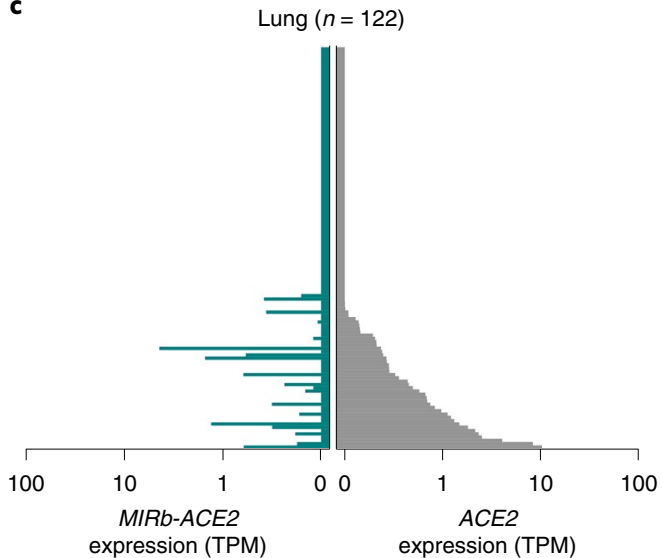

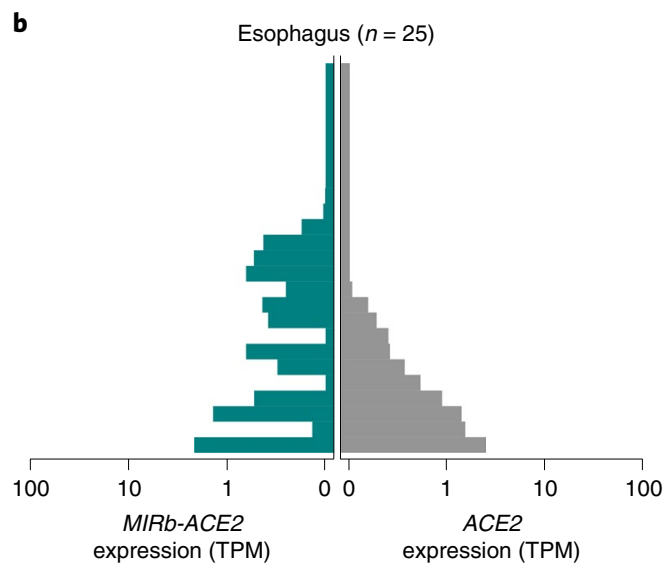

d

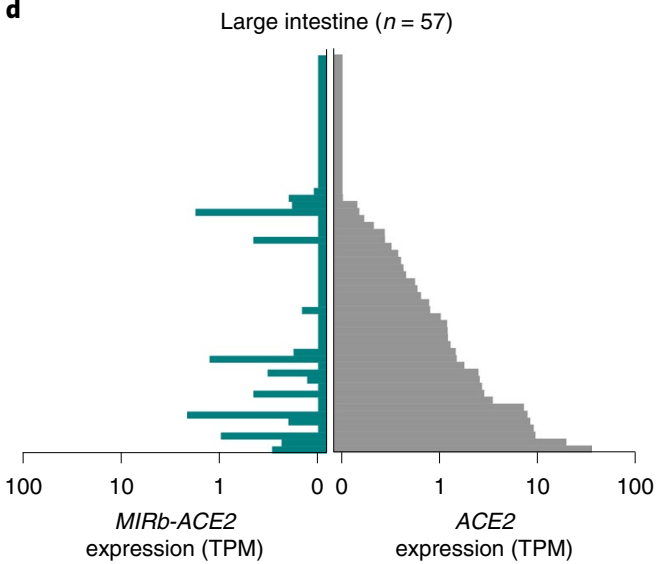

Fig. 3 | ACE2 and MIRb-ACE2 isoform expression in cell lines. a, ACE2 and MIRb-ACE2 isoform expression in cell lines from the upper aerodigestive tract. b, ACE2 and MIRb-ACE2 isoform expression in cell lines from the esophagus. c, ACE2 and MIRb-ACE2 isoform expression in cell lines from the lung. $\mathbf{d}$, ACE2 and MIRb-ACE2 isoform expression in cell lines from the large intestine tract. In a-d, each bar represents an individual sample.

To explain the apparent inefficiency of protein production from $M I R b$-ACE2 transcripts, we cloned the coding sequences of both isoforms into the pcDNA3.1-DYK-P2A-eGFP expression vector, which adds both a FLAG tag and P2A peptide-linked GFP as part of the protein product. Expression of GFP was comparable in $A C E 2$-transfected and MIRb-ACE2-transfected cells, suggesting that the single RNA molecule that encodes for both the FLAG-tagged MIRb-ACE2 product and GFP is stable and translated (Fig. 5a). Despite that, following transfection with plasmid concentrations producing readily detectable full-length ACE2 and resulting in $M I R b$-ACE2 RNA levels comparable with those endogenously produced in IFN- $\alpha$-stimulated cells, we could not detect the predicted MIRb-ACE2 protein with antibodies to the FLAG tag (Fig. 5b). However, the FLAG-tagged MIRb-ACE2 protein could be detected in HEK293T cells transfected with much higher plasmid concentrations, resulting in RNA expression levels which were one order of magnitude higher than those observed in IFN- $\alpha$-stimulated NHBE cells and SCC-4 or SCC-25 cells (Fig. 5c). Therefore, although the $M I R b$-ACE2 transcript was efficiently translated (supported by the levels of P2A-linked GFP), the MIRb-ACE2 protein product was much less abundant than the full-length ACE2 at a given RNA transcription level, suggesting posttranslational protein instability of the former.

Lysine residues 625 and 702 in the full-length ACE2 protein have been described to be ubiquitinated and may contribute to its proteosomal degradation ${ }^{26}$. We generated a K625R K702R (K2R) mutant of full-length ACE2, which increased protein levels, compared to wild-type ACE2 (Fig. 5d). We have introduced the same mutations in the corresponding residues of the predicted MIRb-ACE2 protein product, K279R K356R, which were similarly accessible for ubiquitination (Extended Data Fig. 8). However, we were unable to detect stable protein following transfection with the MIRb-ACE2 K2R-encoding mutant (Fig. 5d). Consistent with this, the addition of the proteasome inhibitor MG-132 was sufficient to increase protein levels of ACE2 but did not rescue the MIRb-ACE2 protein product (Fig. 5d). Moreover, cycloheximide treatment of HEK293T cells transfected with FLAG-tagged ACE2 or MIRb-ACE2 constructs led to the rapid loss of MIRb-ACE2 protein but did not affect levels of full-length ACE2 in the same time frame (Fig. 5e), further supporting reduced stability of the former.

Structural considerations suggested that the MIRb-ACE2 protein product would unlikely retain the partial structure of the canonical ACE2 peptidase fold, as removing most of this subdomain would expose the remaining component of the highly charged substrate-binding groove, as well as large parts of the hydrophobic protein core (Extended Data Fig. 9). Hence, it seems unlikely that a protein encoded by the MIRb-ACE2 transcript would form a structure similar to that of the canonical ACE2. Moreover, the MIRb-ACE2 protein product lacks a canonical signal peptide and when an IgGK chain-derived signal peptide, which has been successfully used to express the canonical ACE2 ectodomain (residues 15-615) 27 $^{2}$, was fused to the corresponding domain of the predicted MIRb-ACE2 protein (residues 1-269), there was no detectable secreted protein. These data suggest that the latter 
a

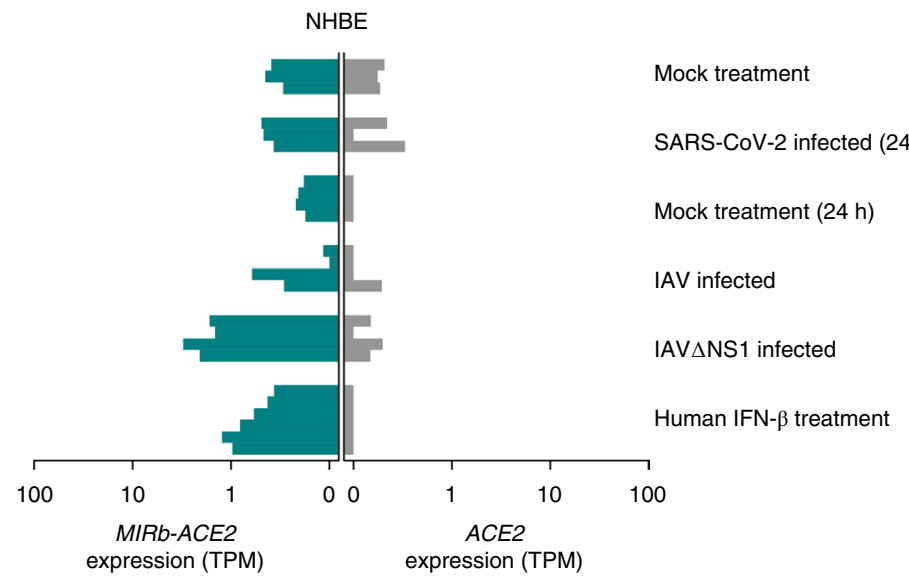

b

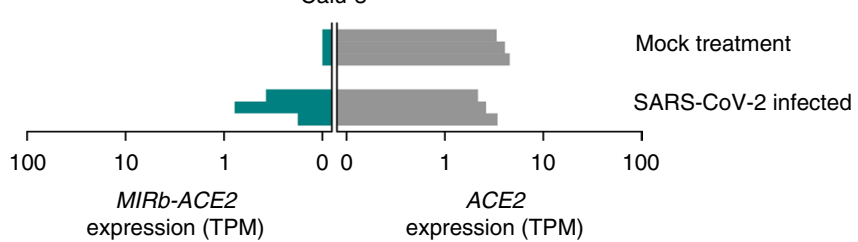

C

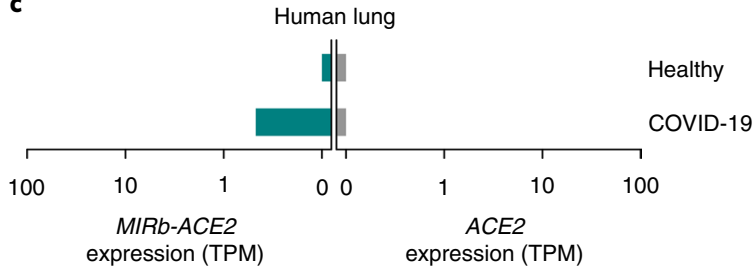

d

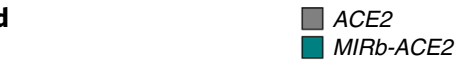

e
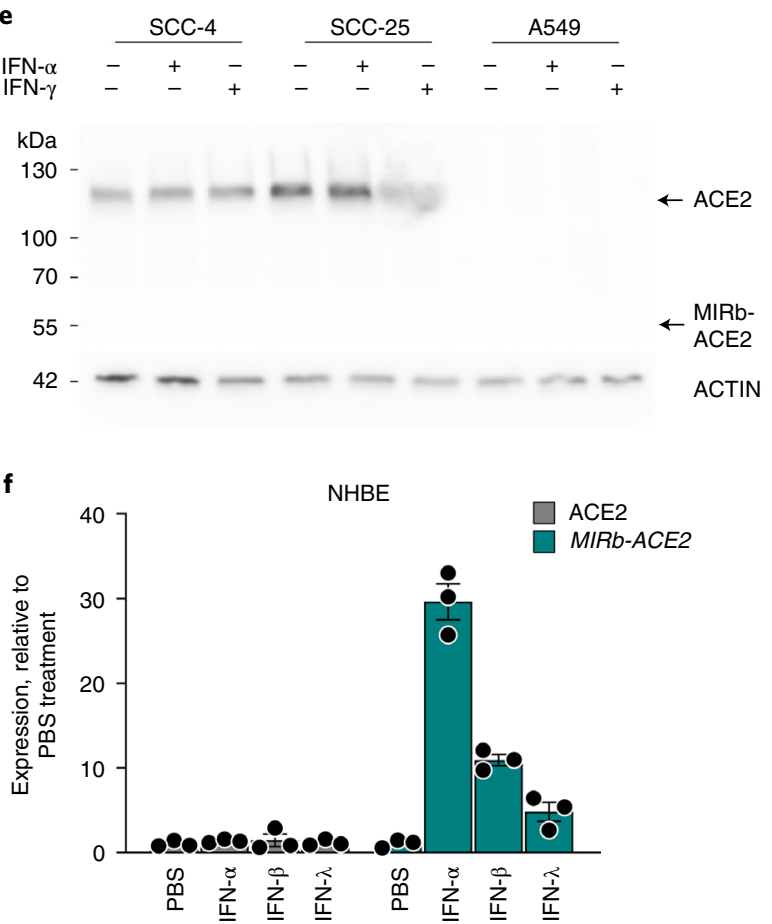

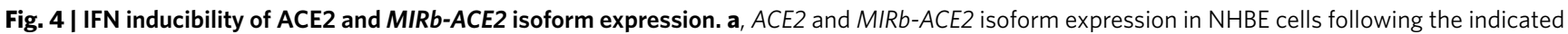
treatment. b, ACE2 and MIRb-ACE2 isoform expression in Calu-3 cells with or without infection with SARS-CoV-2. c, ACE2 and MIRb-ACE2 isoform expression in the lung of a patient with COVID-19 and in a healthy lung. In a-c, raw data were obtained from study GSE147507. Each bar represents an individual sample. d, Mean ( \pm standard error (s.e.)) ACE2 and MIRb-ACE2 isoform expression, determined by RT-qPCR in SCC-4 and SCC-25 cells with or without IFN stimulation. e, Detection of ACE2 and putative MIRb-ACE2 protein product by western blotting in cell lysates from the same cells as in $\mathbf{d}$. One representative experiment of two is shown. f, Mean ( \pm s.e.) ACE2 and MIRb-ACE2 isoform expression, determined by RT-qPCR in NHBE cells with or without IFN stimulation. In $\mathbf{d}$ and $\mathbf{f}$, each symbol represents the mean value of two technical RT-qPCR replicates of a single culture, and the bars and error bars represent the mean and s.e. of the three independently treated cultures in the same experiment.

protein is subject to posttranslational degradation through a proteasome-independent mechanism and thus unlikely to exert significant biological activity.

Nevertheless, as the MIRb-ACE2 protein was indeed made under certain conditions, it remained possible that it retained some biological function or that it affected the function of canonical ACE2 through heterodimer formation. To examine this possibility, we quantified levels of enzymatically active ACE2, an assay that is considerably more sensitive than western blotting, and found that, as expected, strong enzymatic activity in lysates from $A C E 2$-transfected cells but not MIRb-ACE2-transfected cells (Fig. 5f). Furthermore, co-transfection with $M I R b-A C E 2$ did not affect the enzymatic activity conferred by ACE2 transfection (Fig. 5f).
To determine any involvement of the predicted MIRb-ACE2 protein in SARS-CoV-2 entry, we measured binding of the S1 subunit of SARS-CoV-2 spike glycoprotein, the first step of viral entry, to cells expressing either or both ACE2 isoforms. HEK293T cells were transfected with the P2A-GFP reporter constructs for ACE2 and $M I R b-A C E 2$ and transfected and untransfected cells were distinguished based on GFP expression (Extended Data Fig. 10). While SARS-CoV-2 S1 efficiently bound HEK293T cells expressing ACE2, it did not bind those expressing MIRb-ACE2 (Fig. 5g). Moreover, co-expression of the two isoforms in the same cells did not alter binding of SARS-CoV-2 S1, beyond the effect of plasmid dilution (Fig. 5g). Collectively, these results argue against a significant effect of $M I R b$-ACE2 expression on ACE2 function or SARS-CoV-2 entry. 
a

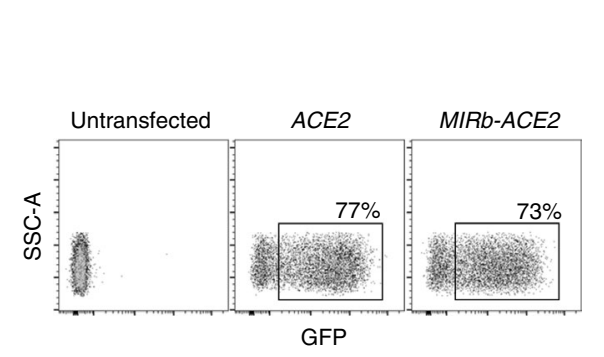

d

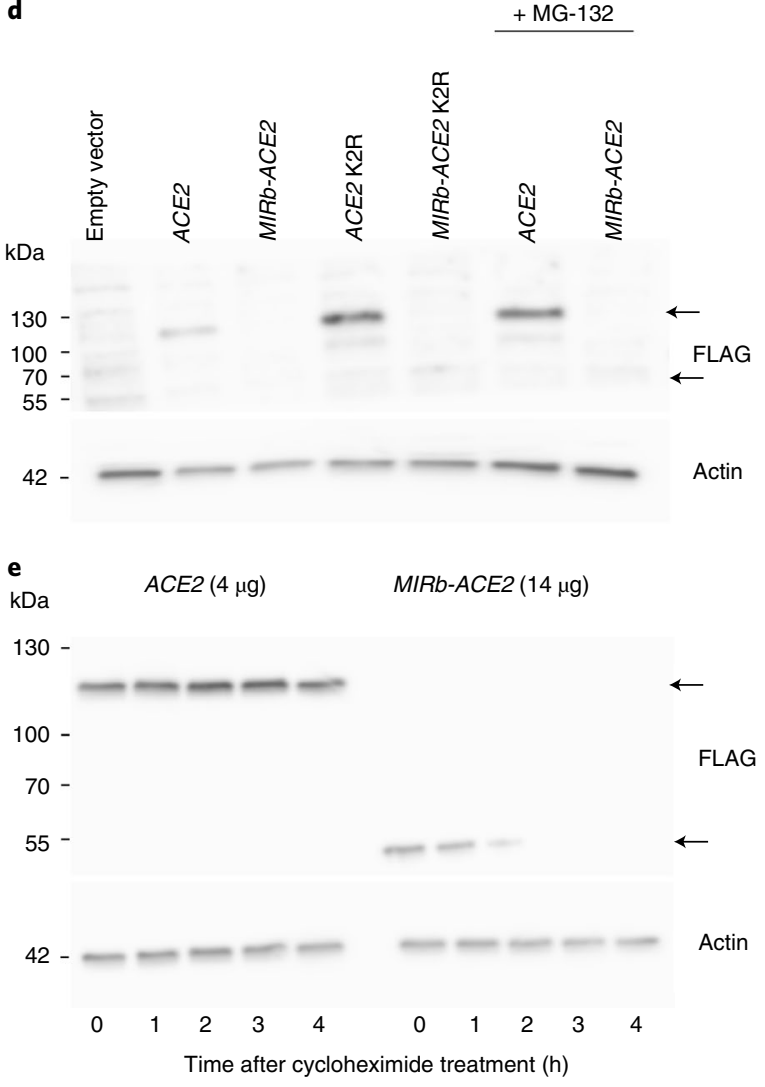

b

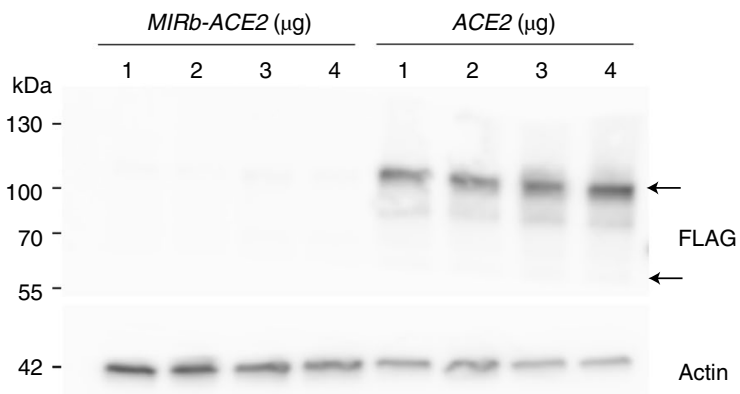

$\mathrm{kDa}$

$100-$

FLAG
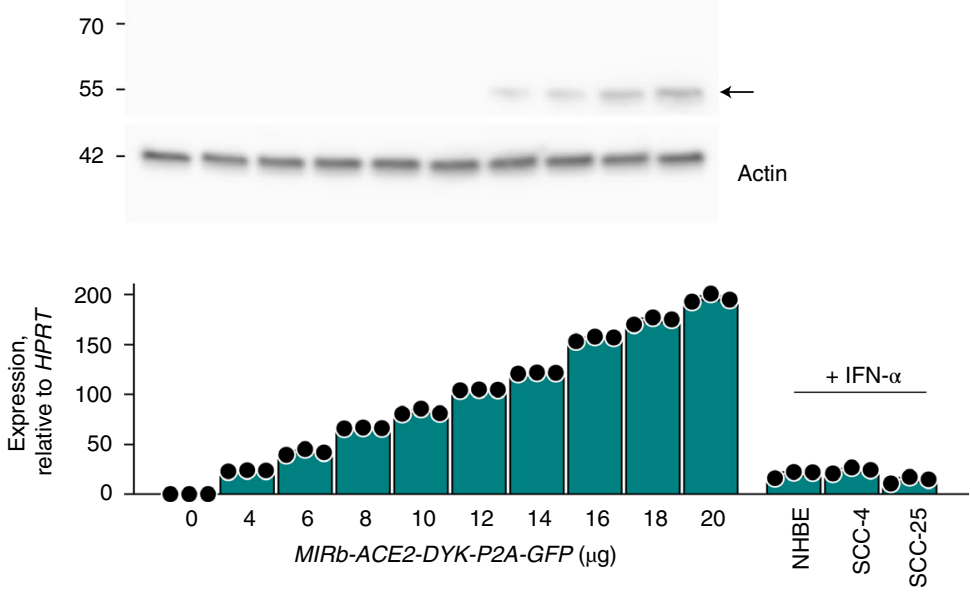

f
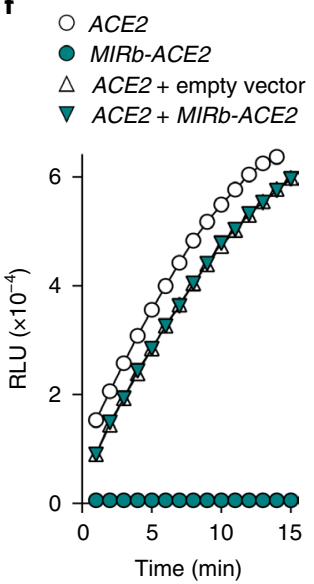

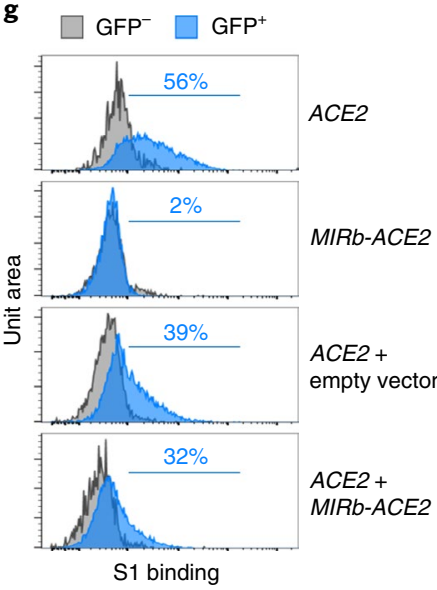

Fig. 5 | Stability of the ACE2 and MIRb-ACE2 translation products. a, Flow cytometric detection of GFP expression (left) and quantification of mean frequency ( \pm s.e.) of GFP-expressing cells (right) in HEK293T cells transfected to express either ACE2 or MIRb-ACE2 in conjunction with a FLAG tag and GFP, linked by a P2A peptide. Symbols represent three independently transfected cultures in the same experiment. One representative experiment of three is shown. $\mathbf{b}$, Detection of ACE2 and putative MIRb-ACE2 protein by western blotting for the FLAG tag in cell lysates from the same cells as in $\mathbf{a}$. Titration of the transfection plasmids used is also indicated. One representative experiment of two is shown. c, Detection of MIRb-ACE2 protein by western blotting for the FLAG tag in HEK293T cells transfected with increasing amounts of the expression plasmid (top) and mean ( \pm s.e.) MIRb-ACE2 expression, determined by RT-qPCR in the same cells, in comparison with MIRb-ACE2 expression in IFN- $\alpha$-stimulated NHBE cells and SCC- 4 and SCC- 25 cells (bottom). Each symbol represents the mean value of two technical RT-qPCR replicates of a single culture, and the bars and error bars represent the mean and s.e. of the three independently treated cultures in the same experiment. d, Detection of ACE2 and MIRb-ACE2 protein by western blotting for the FLAG tag in cell lysates from HEK293T cells transfected (with $4 \mu \mathrm{g}$ of expression plasmids) to express either wild-type isoform or either isoform with the two lysine residues mutated (K2R; all in conjunction with a FLAG tag and GFP, linked by a P2A peptide). HEK293T cells transfected to express the wild-type isoforms were treated with the MG-132 inhibitor. One representative experiment of two is shown. e, Stability of ACE2 and MIRb-ACE2 protein, determined by western blotting in HEK293T cells transfected to express either isoform, after the indicated times following treatment with cycloheximide. Data from a single experiment are shown. f, Kinetics of mean ( \pm s.d.) ACE2 enzymatic activity in the supernatant of HEK293T cells transfected to express either ACE2 or MIRb-ACE2 or both (ACE2 + MIRb-ACE2). Expression plasmids were used at $4 \mu \mathrm{g}$ and $2 \mu \mathrm{g}$ each for individual transfections and co-transfections, respectively. Symbols represent the mean value of two technical replicates in the same experiment. One representative experiment of two is shown. RLU, relative light units. $\mathbf{g}$, Flow cytometric detection of SARS-CoV-2 S1 bindings to HEK293T cells transfected to express either ACE2 or MIRb-ACE2 or both (ACE2 + MIRb-ACE2). ACE2 and MIRb-ACE2 expression plasmids were used at $4 \mu \mathrm{g}$ and $14 \mu \mathrm{g}$ for individual transfections, respectively, and at $2 \mu \mathrm{g}$ and $14 \mu \mathrm{g}$ for co-transfections, respectively. 


\section{Discussion}

Regulation of ACE2 expression and function is critical both in physiology and pathology ${ }^{28}$. The use of ACE2 as a primary receptor for entry by the pandemic coronaviruses SARS-CoV and SARS-CoV-2 highlighted the potential effect of changes in ACE2 expression, particularly in response to IFN, on the course or severity of COVID-19 (ref. ${ }^{17}$ ). Here we show that ACE2 transcription and protein production is not responsive to IFN. Instead, we describe a new RNA isoform, $M I R b-A C E 2$, that is highly responsive to IFN stimulation, but encodes a truncated and unstable protein product. In support of these findings, the new isoform is independently described in two other recent preprint reports ${ }^{24,25}$ and matches the sequence recently deposited under GenBank accession number MT505392. We find that the $M I R b-A C E 2$ isoform exhibits distinct patterns of expression along the aerodigestive and gastrointestinal tracts and was likely responsible for the apparent IFN inducibility of ACE2 expression reported by analysis of scRNA-seq data ${ }^{17}$ and other similar studies ${ }^{20}$ We further show that transcription of this new isoform is initiated by intronic retroelements, which function as a cryptic, IFN-responsive promoter, adding further evidence for the widespread involvement of such retroelements in gene regulatory networks.

Indeed, endogenous retroelements constitute nearly half of the human genome and can affect many host processes ${ }^{29-31}$. LTR and non-LTR retroelements represent an abundant source of promoters, enhancers and polyadenylation sequences that can modulate the expression and structure of neighboring genes ${ }^{32}$, as with ACE2. For instance, retroelements serve as promoters or enhancers for a number of ISGs, conferring IFN inducibility, exemplified in the case of AIM2 ( ref. $^{7}$ ). Retroelements may further modify the function of ISGs, and we have recently described a new isoform of the ISG CD274 (encoding programmed death-ligand 1) that produces a truncated form through retroelement exonization ${ }^{33}$.

The use of the intronic MIRb and LTR16A1 elements as the promoter and alternative exon for the $M I R b-A C E 2$ isoform explains its independent regulation from that of the full-length $A C E 2$ isoform. In addition to IFN inducibility, the cryptic MIRb-LTR16A1 promoter also confers tissue-specific expression, with the highest levels seen in the upper aerodigestive tract, where it can be the predominant isoform. In contrast, the canonical $A C E 2$ isoform far exceeds expression of the $M I R b-A C E 2$ isoform in the lower gastrointestinal tract. It is theoretically possible that the balance of $M I R b-A C E 2$ and full-length ACE2 isoforms plays a role in the spread of SARS-CoV-2, particularly in the upper aerodigestive tract, or that RNA or protein products of $M I R b-A C E 2$ are involved in other pathological or physiological processes. However, the low stability of the MIRb-ACE2 protein product argues that this is unlikely.

Independently of any functional significance, expression of the $M I R b-A C E 2$ isoform needs to be carefully considered in studies examining ACE2 regulation at the transcriptional level $1^{17,19,20}$. The description of this new isoform highlights the need to validate scRNA-seq data with orthogonal approaches. While scRNA-seq initiatives are an invaluable resource and allow for rapid identification of cell types that express a gene of interest, coverage and read depth are largely insufficient to distinguish between isoforms. Technological advances to improve sequencing depth and bioinformatic tools to impute missing values are rapidly progressing; in the meantime, long-read sequencing techniques to quantify transcript isoforms and confirmation of protein expression levels can be incorporated into existing workflows.

This work established $M I R b-A C E 2$ as the predominantly induced form of $A C E 2$ following viral infection or recombinant IFN treatment, including in the SARS-CoV-2-infected lung. The suggestion that ACE2 is an ISG raised fears that therapeutic IFN could be detrimental ${ }^{17}$; however, we find that full-length ACE2 is not increased at the mRNA or protein level. The predicted MIRbACE2 protein product could be detected in vitro, albeit under high levels of $M I R b-A C E 2$ RNA expression, and it remains possible that the MIRb-ACE2 protein, or fragments thereof, are produced under certain conditions in vivo. Indeed, despite its reduced stability when compared to full-length ACE2, evidence for production of the MIRb-ACE2 protein has also been independently reported ${ }^{24,25}$. Nevertheless, it is worth noting that the predicted MIRb-ACE2 protein does not contain the residues required for SARS-CoV-2 spike glycoprotein binding ${ }^{15}$, does not bind recombinant SARS-CoV-2 S1 experimentally and is thus unlikely to contribute to viral spread. These results reconcile the apparent discrepancy between the IFN inducibility of $A C E 2$ with promising data showing improved outcomes in COVID-19 following IFN treatment ${ }^{4,5}$.

\section{Online content}

Any methods, additional references, Nature Research reporting summaries, source data, extended data, supplementary information, acknowledgements, peer review information; details of author contributions and competing interests; and statements of data and code availability are available at https://doi.org/10.1038/ s41588-020-00732-8.

Received: 24 July 2020; Accepted: 29 September 2020; Published online: 19 October 2020

\section{References}

1. Sadler, A. J. \& Williams, B. R. Interferon-inducible antiviral effectors. Nat. Rev. Immunol. 8, 559-568 (2008).

2. Stetson, D. B. \& Medzhitov, R. Type I interferons in host defense. Immunity 25, 373-381 (2006).

3. Gibbert, K., Schlaak, J. F., Yang, D. \& Dittmer, U. IFN- $\alpha$ subtypes distinct biological activities in anti-viral therapy. Br. J. Pharmacol. 168, 1048-1058 (2013).

4. Hung, I. F. et al. Triple combination of interferon beta-1b, lopinavir-ritonavir and ribavirin in the treatment of patients admitted to hospital with COVID-19: an open-label, randomised, phase 2 trial. Lancet 395, 1695-1704 (2020).

5. Wang, N. et al. Retrospective multicenter cohort study shows early interferon therapy is associated with favorable clinical responses in COVID-19 patients. Cell Host Microbe https://doi.org/10.1016/j.chom.2020.07.005 (2020).

6. Ivashkiv, L. B. \& Donlin, L. T. Regulation of type I interferon responses. Nat. Rev. Immunol. 14, 36-49 (2014).

7. Chuong, E. B., Elde, N. C. \& Feschotte, C. Regulatory evolution of innate immunity through co-option of endogenous retroviruses. Science 351, 1083-1087 (2016).

8. Young, G. R. et al. Resurrection of endogenous retroviruses in antibody-deficient mice. Nature 491, 774-778 (2012).

9. Young, G. R., Mavrommatis, B. \& Kassiotis, G. Microarray analysis reveals global modulation of endogenous retroelement transcription by microbes. Retrovirology 11, 59 (2014).

10. Attig, J., Young, G. R., Stoye, J. P. \& Kassiotis, G. Physiological and pathological transcriptional activation of endogenous retroelements assessed by RNA-sequencing of B lymphocytes. Front. Microbiol. 8, 2489 (2017).

11. Tokuyama, M. et al. ERVmap analysis reveals genome-wide transcription of human endogenous retroviruses. Proc. Natl Acad. Sci. USA 115, 12565-12572 (2018).

12. García-Sastre, A. Ten Strategies of interferon evasion by viruses. Cell Host Microbe 22, 176-184 (2017).

13. Blanco-Melo, D. et al. Imbalanced host response to SARS-CoV-2 drives development of COVID-19. Cell 181, 1036-1045 (2020).

14. Kopecky-Bromberg, S. A., Martínez-Sobrido, L., Frieman, M., Baric, R. A. \& Palese, P. Severe acute respiratory syndrome coronavirus open reading frame (ORF) 3b, ORF 6 and nucleocapsid proteins function as interferon antagonists. J. Virol. 81, 548-557 (2007).

15. Shang, J. et al. Structural basis of receptor recognition by SARS-CoV-2. Nature 581, 221-224 (2020).

16. Hoffmann, M. et al. SARS-CoV-2 cell entry depends on ACE2 and TMPRSS2 and is blocked by a clinically proven protease inhibitor. Cell 181, 271-280 (2020).

17. Ziegler, C. G. K. et al. SARS-CoV-2 receptor ACE2 is an interferon-stimulated gene in human airway epithelial cells and is detected in specific cell subsets across tissues. Cell 181, 1016-1035 (2020).

18. Attig, J. et al. LTR retroelement expansion of the human cancer transcriptome and immunopeptidome revealed by de novo transcript assembly. Genome Res. 29, 1578-1590 (2019).

19. Singh, M., Bansal, V. \& Feschotte, C. A single-cell RNA expression map of human coronavirus entry factors. Cell Rep. 32, 108175 (2020). 
20. Smith, J. C. et al. Cigarette smoke exposure and inflammatory signaling increase the expression of the SARS-CoV-2 receptor ACE2 in the respiratory tract. Dev. Cell 53, 514-529 (2020).

21. Han, X. et al. Construction of a human cell landscape at single-cell level. Nature 581, 303-309 (2020).

22. Major, J. et al. Type I and III interferons disrupt lung epithelial repair during recovery from viral infection. Science https://doi.org/10.1126/science.abc2061 (2020).

23. Ng, K. W. et al. Pre-existing and de novo humoral immunity to SARS-CoV-2 in humans. Preprint at bioRxiv https://doi.org/10.1101/2020.05.14.095414 (2020).

24. Onabajo, O. O. et al. Interferons and viruses induce a novel truncated ACE2 isoform and not the full-length SARS-CoV-2 receptor. Nat. Genet. https://doi. org/10.1038/s41588-020-00731-9 (2020).

25. Blume, C. et al. A novel isoform of ACE2 is expressed in human nasal and bronchial respiratory epithelia and is upregulated in response to RNA respiratory virus infection. Preprint at bioRxiv https://doi. org/10.1101/2020.07.31.230870 (2020).

26. Stukalov, A. et al. Multi-level proteomics reveals host-perturbation strategies of SARS-CoV-2 and SARS-CoV. Preprint at bioRxiv https://doi. org/10.1101/2020.06.17.156455 (2020).
27. Wrobel, A. G. et al. SARS-CoV-2 and bat RaTG13 spike glycoprotein structures inform on virus evolution and furin-cleavage effects. Nat. Struct. Mol. Biol. https://doi.org/10.1038/s41594-020-0468-7 (2020).

28. Hamming, I. et al. The emerging role of ACE2 in physiology and disease. J. Pathol. 212, 1-11 (2007).

29. Burns, K. H. \& Boeke, J. D. Human transposon tectonics. Cell 149 , 740-752 (2012).

30. Feschotte, C. \& Gilbert, C. Endogenous viruses: insights into viral evolution and impact on host biology. Nat. Rev. Genet. 13, 283-296 (2012).

31. Kassiotis, G. \& Stoye, J. P. Immune responses to endogenous retroelements taking the bad with the good. Nat. Rev. Immunol. 16, 207-219 (2016).

32. Thompson, P. J., Macfarlan, T. S. \& Lorincz, M. C. Long terminal repeats: from parasitic elements to building blocks of the transcriptional regulatory repertoire. Mol. cell 62, 766-776 (2016).

33. Ng, K. W. et al. Soluble PD-L1 generated by endogenous retroelement exaptation is a receptor antagonist. eLife $\mathbf{8}$ https://doi.org/10.7554/eLife.50256 (2019)

Publisher's note Springer Nature remains neutral with regard to jurisdictional claims in published maps and institutional affiliations.

(c) The Author(s), under exclusive licence to Springer Nature America, Inc. 2020 


\section{Methods}

Cell lines. HEK293T, A549, SCC-4, SCC-25, Vero, CV-1, MDCK, R9ab and MCA-38 cells were obtained from and verified as mycoplasma free by the Cell Services facility at the Francis Crick Institute. Human cell lines were additionally validated by DNA fingerprinting. HEK293T and A549 cells were grown in IMDM (Sigma-Aldrich) supplemented with $5 \%$ FBS (Thermo Fisher Scientific), L-glutamine ( $2 \mathrm{mmol}^{-1}$; Thermo Fisher Scientific), penicillin (100 $\mathrm{U} \mathrm{ml}^{-1}$; Thermo Fisher Scientific) and streptomycin $\left(0.1 \mathrm{mg} \mathrm{ml}^{-1}\right.$; Thermo Fisher Scientific). SCC-4 and SCC-25 cells were grown in DMEM with nutrient mixture F-12 (Gibco) supplemented with 10\% FBS (Thermo Fisher Scientific), L-glutamine $\left(2 \mathrm{mmol}^{-1}\right.$; Thermo Fisher Scientific), penicillin $\left(100 \mathrm{U} \mathrm{ml}^{-1}\right.$; Thermo Fisher Scientific) and streptomycin $\left(0.1 \mathrm{mg} \mathrm{ml}^{-1}\right.$; Thermo Fisher Scientific). NHBE cells were cultured as previously described ${ }^{22}$.

Transcript identification, read mapping and quantification. Transcripts were previously assembled on a subset of the RNA-seq data from TCGA ${ }^{18}$. The alternative promoter within ACE2 was more highly expressed in lung squamous carcinomas than the canonical isoform, prompting us to investigate its biology. RNA-seq data from TCGA, GTEx, CCLE and other studies were mapped to the cancer-tissue transcriptome assembly and counted as previously described ${ }^{18}$. Briefly, TPM values were calculated for all transcripts in the transcript assembly ${ }^{18}$ with a custom Bash pipeline (Supplementary Code 1) using GNU paralle ${ }^{34} \mathrm{v} 3$ and Salmon ${ }^{35} \mathrm{v} 0.12 .0$, which uses a probabilistic model for assigning reads aligning to multiple transcript isoforms, based on the abundance of reads unique to each isoform ${ }^{35}$. Splice junctions were visualized using the Integrative Genome Viewer ${ }^{36}$ v2.4.19.

Additional bulk RNA-seq and scRNA-seq data analysis. Bulk RNA-seq data were downloaded from study GSE147507 (ref. ${ }^{13}$ ). Reads were adaptor trimmed and filtered for minimal 35-nucleotide long sequences using Trimmomatic v0.39. Since some samples were infected with SARS-CoV-2 in vitro, we identified and removed viral reads using BowTie2 (seed length: 30 nucleotides) to align reads to the Wuhan region reference genome (MN908947). Subsequently, reads were mapped with HISAT2 (optional parameters: --p 8 --q --k 5) against GRCh38 reference chromosome assembly, and transcripts were quantified against our custom transcriptome assembly using Salmon ${ }^{35} \mathrm{v} 0.12 .0$, as described previously ${ }^{18}$ and in Transcript identification, read mapping and quantitation.

For scRNA-seq data analysis, we downloaded the raw paired-end sequencing reads as unmapped bam files from study GSE134355 (ref. ${ }^{21}$ ), which were already demultiplexed, with one individual per tissue for each sample. We then used the DropSeq Picard toolbox (v2.3.0) to recapitulate processing of human cell landscape (HCL) samples as documented on https://github.com/ggjlab/HCL. In summary, this includes trimming polyA ends from each primary RNA-sequencing read and tagging it with the cellular and molecular adaptor sequence contained in the secondary read (BASE_RANGE $=1-6: 22-27: 43-48$ and BASE_RANGE $=49-54$, respectively). All reads were then mapped with HISAT2 (optional parameters --p 8 --q --k 5) against GRCh38 reference chromosome assembly. The HISAT2 index here was built with the --exon/--ss option to cover all known splice sites annotated in the GENCODE v34 basic annotation. The cellular and molecular barcode sequences were recovered using the MergeBamAlignment utility in Picard.

5'RACE PCR and sequencing. Total RNA from NHBE, SCC-4 and SCC-25 cells was isolated using the QIAcube (Qiagen), and cDNA synthesis was carried out using the High Capacity Reverse Transcription Kit (Applied Biosystems), with an added RNase inhibitor (Promega). Amplicons were generated using the 5' RACE System (Invitrogen), according to the manufacturer's instructions using primers listed in Supplementary Table 1. Libraries were prepared from amplicons using the NEB Ultra II DNA Library Prep Kit for Illumina (New England Biolabs), according to the manufacturer's instructions and sequenced on a MiSeq system (Illumina). Reads were quality and adaptor trimmed in pairs using cutadap ${ }^{37} \mathrm{v} 1.18$ and aligned with $\mathrm{STAR}^{38} \mathrm{v} 2.7 .1 \mathrm{a}$ (settings: outFilterScoreMinOverLread $=0.1$ and outFilterMatchNminOverLread $=0.1$ ) to the a GRCh38 reference with known slice sites from Ensembl release 100. The most 5' base of reads mapping to the $M I R b-A C E 2$ transcript was taken as the transcription start site and was obtained from the properly paired, uniquely mapping reads using bedtools for visualization within Integrative Genome Viewer v2.4.19.

$M I R b$ and $L T R 16 A 1$ sequence alignments. To identify the integration time of LTR16A1 into the ACE2 locus, we first compared the Homo sapiens LTR16A1 and $M I R b$ to the respective consensus sequences in Dfam ${ }^{39}$. Based on sequence identity and the human neutral substitution rate, estimated at $2.2 \times 10^{-9}$ substitutions per site per year, the LTR16A1 insertion is expected to be $\sim 131$ million years (with 284 nucleotide matches across 399 nucleotides) and the MIRb insertion 155 million years (with 159 nucleotide matches across 241 nucleotides). To find evidence for insertion of the LTR16Al and MIRb elements before the split of the major mammalian lineages, we used the The University of California, Santa Cruz (UCSC) LiftOver utility to find the ACE2 gene locus in Rhesus macaque (rheMac10 assembly), marmoset (caljac3 assembly), mouse ( $\mathrm{mm} 10$ assembly), dog (canFam3 assembly), African elephant (loxAfr3 assembly), bottlenose dolphin (Turtru2 assembly), cow (bosTau9 assembly), opossum (monDom5 assembly) and platypus (ornAna2). We used the MUSCLE aligner on default settings to build a global alignment of human to rhesus macaque and marmoset, and then aligned all other species to the profile, reverting the strand of the whole sequence for mouse, elephant, cow and opossum due to whole-gene inversions. We then used the MUSCLE --refine parameter on overlapping 30,000 column blocks to refine the alignment locally. Next, we identified the longest potential sequences matching the LTR16A1 and $M I R b$ elements in all species based on the sequences aligning with the repeat sequence in the human genome as annotated by RepeatMasker. These were aligned to LTR16A1 and MIRb consensus sequences from Dfam 3.2 with mafft (options: --ep 0 --genafpair --maxiterate 1000) and intronic sequences clearly distinct from the repeats were trimmed. The two elements are absent from the considerably shorter platypus ACE2 intron. In opossum, the respective intronic sequence is extended but no clear matches with either LTR16A1 or MIRb were found, prompting us to place both insertions ahead of the mammalian radial divergence. The illustration of the lineage tree including node times is taken from www.timetree.org.

Expression vectors. Open reading frames encoding ACE2, MIRb-ACE2 and respective lysine mutants were synthesized and cloned into the pcDNA3.1-DYK-P2A-eGFP mammalian expression vector. Gene synthesis, cloning and mutagenesis were performed by GenScript and verified by sequencing. Cells were transfected using GeneJuice (EMD Millipore) and harvested $48 \mathrm{~h}$ after transfection for downstream assays.

Cell stimulation. For IFN stimulation experiments, $2 \times 10^{5}$ SCC- 4 and SCC- 25 cells were stimulated with $100 \mathrm{ng} \mathrm{ml}^{-1}$ IFN- $\alpha$ or IFN- $\gamma$ (Abcam) or PBS for $48 \mathrm{~h}$. For proteasome inhibition experiments, cells were cultured in $20 \mu \mathrm{M}$ MG-132 (EMD Millipore) $24 \mathrm{~h}$ after transfection and harvested $48 \mathrm{~h}$ after transfection. For cycloheximide experiments, cells were treated with $250 \mu \mathrm{g} \mathrm{ml}^{-1}$ cycloheximide (Sigma-Aldrich) and harvested at denoted time points. NHBE cells were stimulated for $4 \mathrm{~h}$ with $1,000 \mathrm{ng} \mathrm{ml}^{-1} \mathrm{IFN}-\alpha, 100 \mathrm{ng} \mathrm{ml}^{-1} \mathrm{IFN}-\beta$ or $100 \mathrm{ng} \mathrm{ml}^{-1} \mathrm{IFN}-\lambda$, as used in a previous study ${ }^{22}$, and stored cDNA was analyzed by RT-qPCR in this study.

Western blot. Cell lysates in RIPA buffer were resuspended in SDS buffer, heat denatured at $95^{\circ} \mathrm{C}$ for $10 \mathrm{~min}$, run on a $4-20 \%$ gel (Bio-Rad), transferred to a PVDF membrane (Bio-Rad) and blocked in 5\% (wt/vol) bovine serum albumin fraction V (Sigma-Aldrich) in TBS-T. Membranes were incubated with primary antibodies to ACE2 (1:1,000 dilution; ab15348, Abcam), FLAG (1:1,000; F180450UG clone M2, Sigma-Aldrich), HRP-conjugated secondary antibodies to rabbit IgG or mouse IgG (1:1,000; nos. 7074 and 7076, respectively, Cell Signaling Technology) and HRP-conjugated actin (1:25,000; ab49900, Abcam). Blots were visualized by chemiluminescence on an Amersham Imager 600 (GE Healthcare).

Quantitative PCR with reverse transcription. Total RNA from cell lines was isolated using the QIAcube (Qiagen), and cDNA synthesis was carried out with the High Capacity Reverse Transcription Kit (Applied Biosystems) with an added RNase inhibitor (Promega). Purified cDNA was used to quantify human ACE2 and MIRb-ACE2, or Ace2 and MIRb-Ace2 in other mammalian species, using variant-specific and species-specific primers (Supplementary Table 1). The IFN-inducible human genes CXCL10 and CD274 were also amplified as controls for the effect of IFN treatment, using transcript-specific primers (Supplementary Table 1). For amplification of a conserved housekeeping gene, primers complementary to HPRT sequences conserved in all species were used (Supplementary Table 1). Values were normalized to HPRT expression using the $\Delta \mathrm{C}_{\mathrm{T}}$ method.

Enzyme assays. ACE2 activity in cell lysates was measured using the SensoLyte 390 ACE2 Activity Assay (AnaSpec) according to manufacturer's instructions. Recombinant human ACE2 (Sigma-Aldrich) was used as a positive control.

Flow cytometry. For SARS-CoV-2 S1 binding assays, cells were stained with biotinylated S1 (1:200; Acro Biosystems) for $30 \mathrm{~min}$ followed by APC-Streptavidin (1:200; BioLegend). For S1 binding assays and for GFP detection, single-cell suspensions were run on an LSR Fortessa (BD Biosciences) using BD FACSDiva v8.0 and analyzed with FlowJo v10 (Tree Star) analysis software.

Statistical analysis. Statistical comparisons were made using Prism 7 (GraphPad) or SigmaPlot 14.0. Parametric comparisons of normally distributed values that satisfied the variance criteria were made by unpaired Student's $t$-tests or one-way analysis of variance (ANOVA). Data that did not pass the variance test were compared with non-parametric two-tailed Mann-Whitney rank-sum tests or ANOVA on ranks tests.

Reporting Summary. Further information on research design is available in the Nature Research Reporting Summary linked to this article.

\section{Data availability}

Data supporting the findings of this study are available within the article and its Supplementary Information files. All data, plasmids and cell lines are available from the corresponding author upon reasonable request. Publicly available data were downloaded from the following databases: TCGA Research Network (https:// cancergenome.nih.gov/), The GTEx Project (https://gtexportal.org/home/); and 
the Broad Institute CCLE consortium (https://portals.broadinstitute.org/ccle/). Additionally, RNA-seq data from individual studies (GSE147507 and GSE134355) were downloaded from the Gene Expression Omnibus database (https://www.ncbi. nlm.nih.gov/geo/). Source data are provided with this paper.

\section{Code availability}

Custom code used in this study is available in the Supplementary Information.

\section{References}

34. Tange, O. GNU parallel: the command-line power tool. USENIX Mag. 36, 42-47 (2011).

35. Patro, R., Duggal, G., Love, M. I., Irizarry, R. A. \& Kingsford, C. Salmon provides fast and bias-aware quantification of transcript expression. Nat. Methods 14, 417-419 (2017)

36. Thorvaldsdóttir, H., Robinson, J. T. \& Mesirov, J. P. Integrative Genomics Viewer: high-performance genomics data visualization and exploration. Brief. Bioinform. 14, 178-192 (2013).

37. Marcel, M. Cutadapt removes adapter sequences from high-throughput sequencing reads. EMBnet J. 17, 3 (2011).

38. Dobin, A. et al. STAR: ultrafast universal RNA-seq aligner. Bioinformatics 29, 15-21 (2013).

39. Hubley, R. et al. The Dfam database of repetitive DNA families. Nucleic Acids Res. 44, D81-D89 (2016).

\section{Acknowledgements}

We are grateful for assistance from the Advanced Sequencing, Scientific Computing, Flow Cytometry and Cell Services facilities at the Francis Crick Institute. The results shown here are in whole or in part based upon data generated by TCGA Research Network (https://cancergenome.nih.gov/). The GTEx Project was supported by the Common Fund of the Office of the Director of the National Institutes of Health, and by NCI, NHGRI, NHLBI, NIDA, NIMH and NINDS. This work benefited from data assembled by the CCLE consortium. This work was supported by the Wellcome Trust (102898/B/13/Z) and the Francis Crick Institute (FC001099, FC001206 and FC001078), which receives its core funding from Cancer Research UK, the UK Medical Research Council and the Wellcome Trust.

\section{Author contributions}

K.W.N., J.A., G.R.Y. and G.K. conceived and designed the study. K.W.N., J.A., W.B., G.R.Y., J.M. and A.G.W. performed the experiments. K.W.N., J.A., W.B., G.R.Y., J.M., A.G.W. and G.K. analyzed the data. K.W.N. and G.K. wrote the manuscript with contributions from J.A., W.B., G.R.Y., J.M., A.G.W., S.G. and A.W. All authors approved the final manuscript.

\section{Competing interests}

The authors declare no competing interests.

\section{Additional information}

Extended data is available for this paper at https://doi.org/10.1038/s41588-020-00732-8. Supplementary information is available for this paper at https://doi.org/10.1038/ s41588-020-00732-8.

Correspondence and requests for materials should be addressed to G.K.

Reprints and permissions information is available at www.nature.com/reprints. 


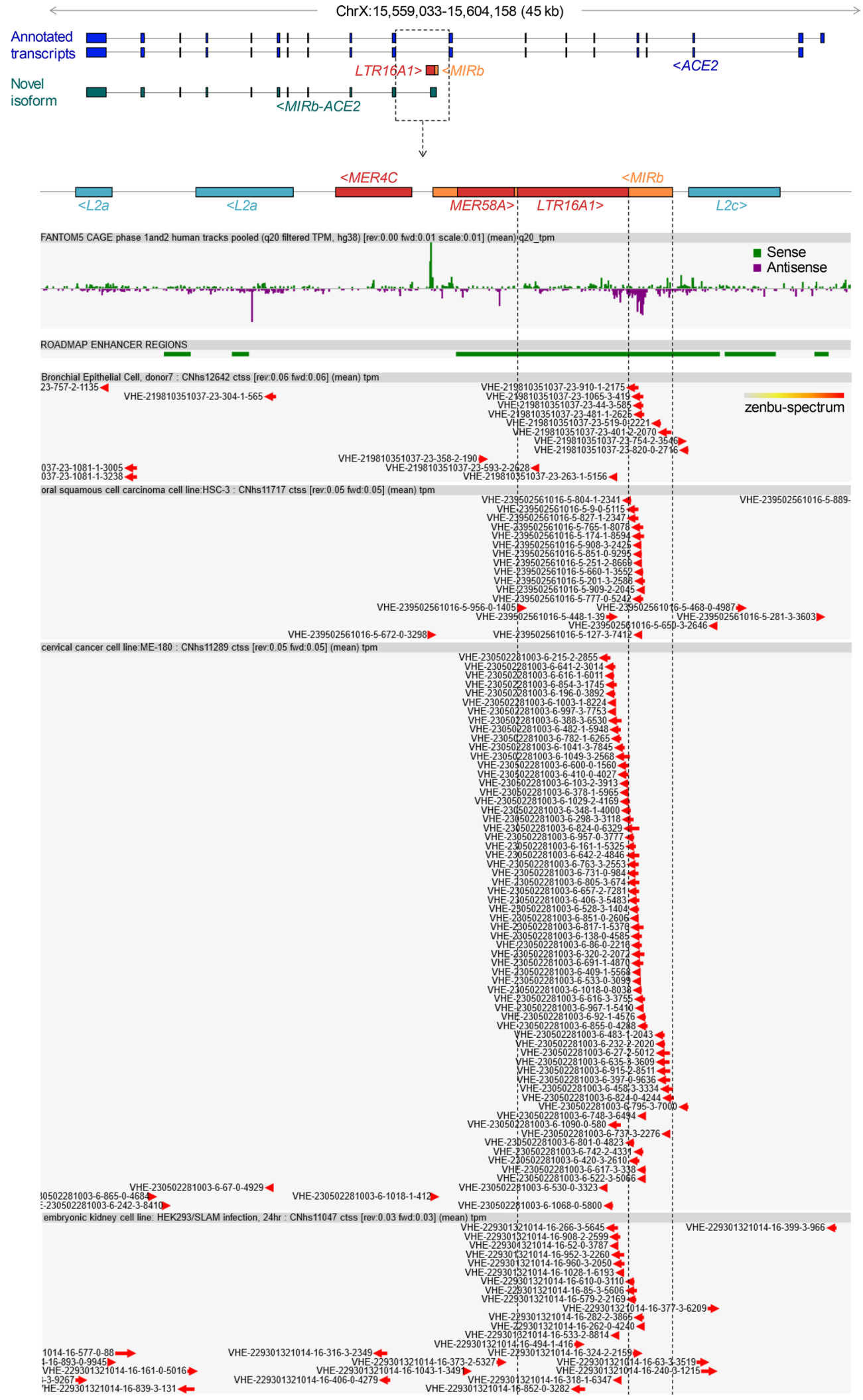

Extended Data Fig. 1 | CAGE support for transcriptional initiation of the MIRb-ACE2 transcript. Normalized data from the FANTOM Consortium and the RIKEN PMI and CLST (DGT) for transcription start sites in the proximity of the intronic MIRb and LTR16A1 elements in the ACE2 locus. Both the sense and antisense orientations are depicted. Data were visualized with the zenbu online viewer (https://fantom.gsc.riken.jp/zenbu) for FANTOM5 Human hg38 promoterome. 
ChrX:15,559,033-15,604,158 (45 kb)

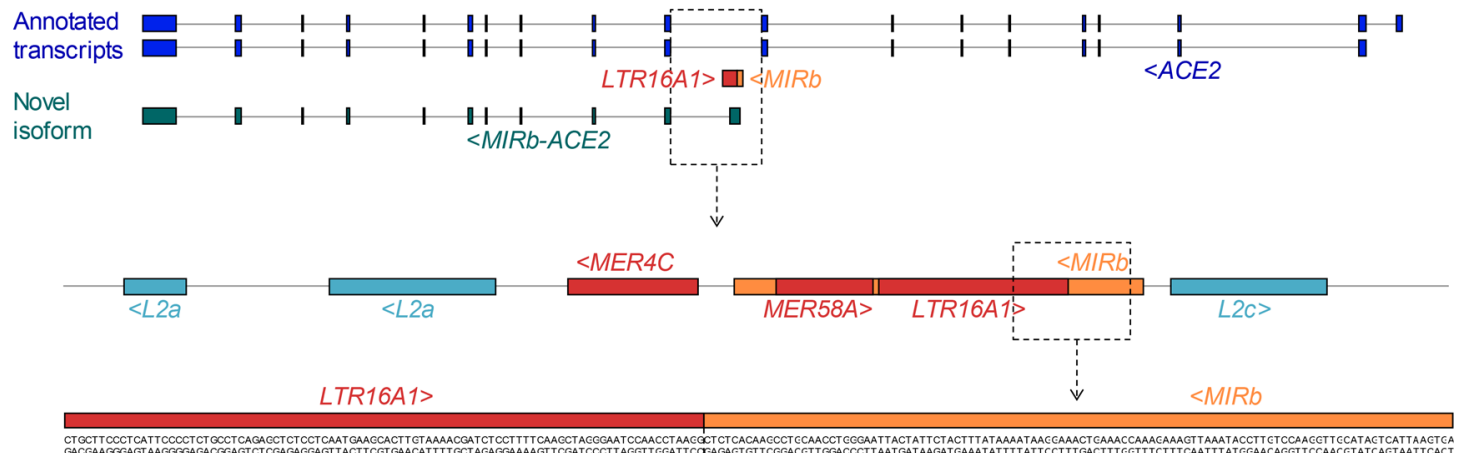

Transcription factor binding sites and putative TATA-box

$6 \quad 0$

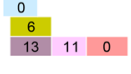

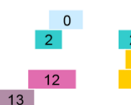

2
9
9

11

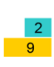

(1) $6^{2}$

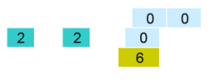

10

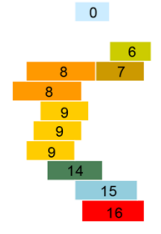

20

\begin{tabular}{ll}
\hline 0 & GR-beta [T01920] \\
1 & XBP-1 [T00902] \\
2 & C/EBPbeta [T00581] \\
\hline 3 & HNF-1A [T00368] \\
4 & HNF-1B [T01950] \\
\hline 5 & LEF-1 [T02905] \\
\hline 6 & STAT4 [T01577]
\end{tabular}

0 GR-beta [T01920]

2 C/EBPbeta [T00581]

4 HNF-1B [T01950]

6 STAT4 [T01577]
7 c-Ets-1 [T00112]

8 TBP [T00794]

9 TFIID [T0082GO]

10 AP-2alphaA [T00035]

11 GR-alpha [T00337]

12 C/EBPalpha [T00105]

13 TFII-I [TO0824]

\section{s}




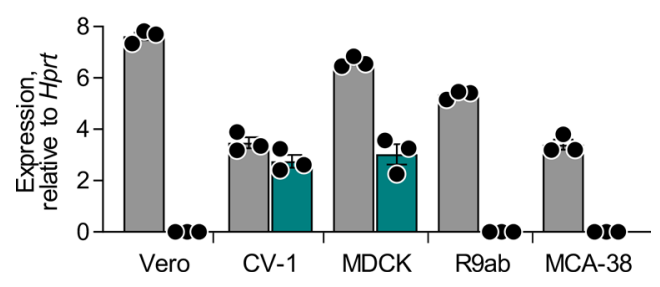

Extended Data Fig. 3 | Ace2 and MIRb-Ace2 expression in cells from representative mammalian species. Mean ( \pm SE) Ace2 and MIRb-Ace2 isoform expression, determined by RT-qPCR, in Vero and CV-1 cells (both from African green monkey), and in MDCK (dog), R9ab (rabbit) and MCA-38 cells (mouse). Expression is normalized to expression of Hprt in each sample. Each symbol represents the mean value of two technical RT-qPCR replicates of a single culture, and the bars and error bars represent the mean and SE of the three individually-treated cultures in the same experiment. 


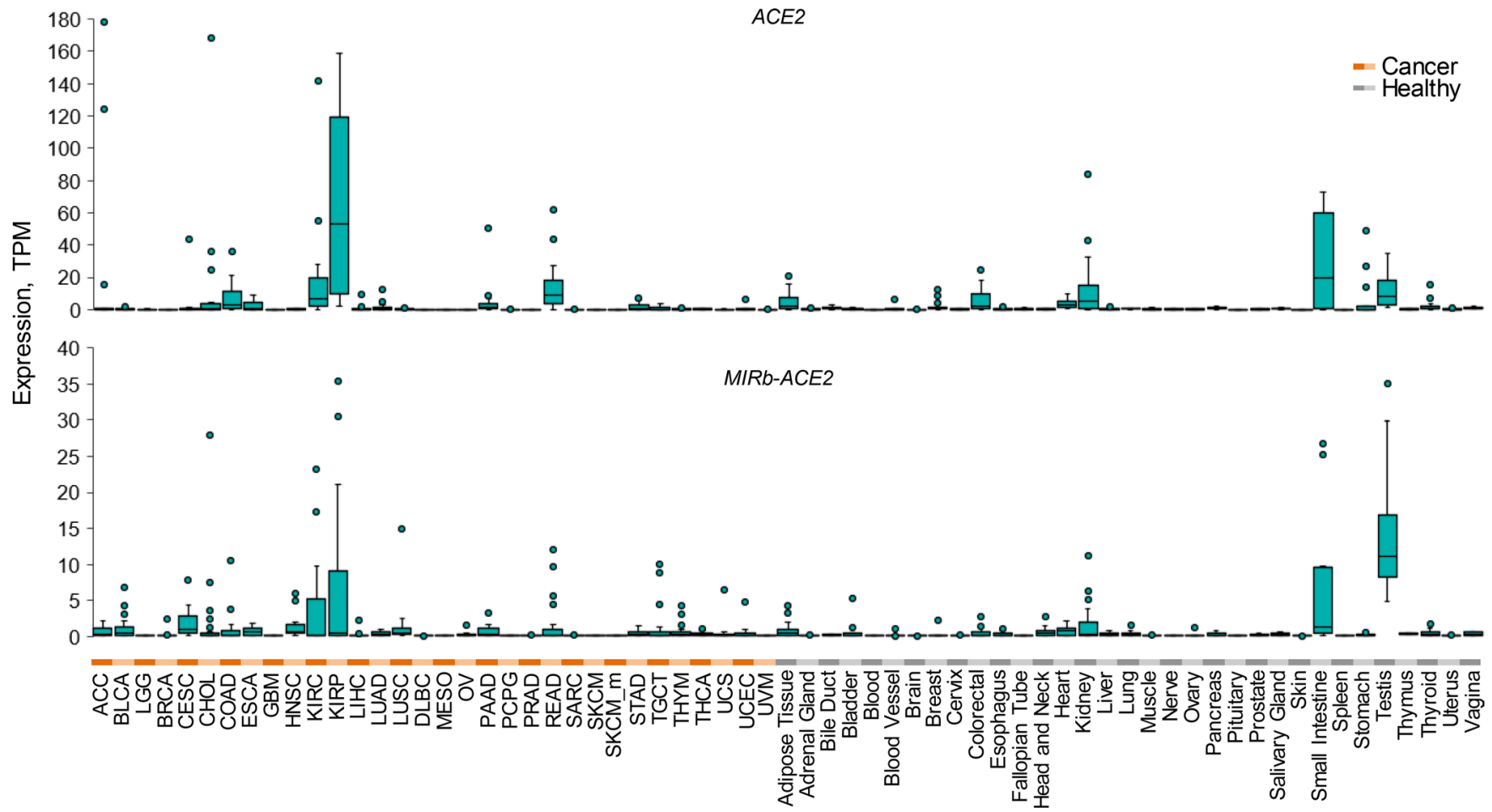

Extended Data Fig. 4 | Expression of ACE2 and MIRb-ACE2 isoforms in cancer and healthy tissues. Box plots of ACE2 and MIRb-ACE2 isoforms expression in cancer patient and healthy control samples from TCGA and GTEx. For each cancer type, 24 samples were included (a total 768 samples), whereas for respective healthy tissues a total of 813 samples were included, varying between 2 and 156 per tissue type. Box plots show the upper and lower quartiles, center lines show the median, whiskers represent the $1.5 x$ interquartile range and individual points represent outliers. 


\section{NATURE GENETICS}

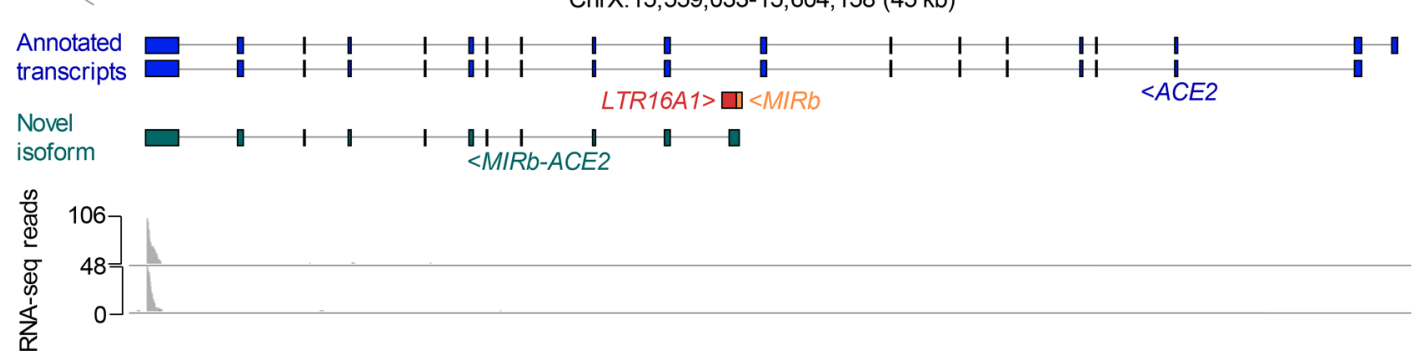

Extended Data Fig. 5 | Single-cell RNA-seq coverage of the ACE2 locus. RNA-seq trace of two multiplexed samples from adult lung, obtained from study GSE134355. Note the lack of coverage across the entire locus with the exception of only the 3 ' end of the last exon, shared between the isoforms. 
ACE2

MIRb-ACE2

ACE2

MIRb-ACE2

ACE2

$\mathrm{MIRB}-\mathrm{ACE} 2$

ACE2

MIRb-ACE2

ACE2

MIRb-ACE2

ACE2

$\mathrm{MIRb}-\mathrm{ACE} 2$

ACE 2

$\mathrm{MIRb}-\mathrm{ACE} 2$

ACE2

MIRb-ACE2

ACE2

MIRb-ACE2

ACE2

MIRb-ACE2
1 MSSSSWLLLSLVAVTAAQSTIEEQAKTFLDKFNHEAEDLFYQSSLASWNYNTNITEENVQNMNNAGDKWSAFLKEQSTLAOMYPL 85

86 QEIQNLTVKLQLQALQQNGSSVLSEDKSKRLNTILNTMSTIYSTGKVCNPDNPQECLLLEPGLNEIMANSLDYNERLWAWESWRS 170 Q

171 EVGKQLRPLYEEYVVLKNEMARANHYEDYGDYWRGDYEVNGVDGYDYSRGQLIEDVEHTFEEIKPLYEHLHAYVRAKLMNAYPSY 255 - - - - - - - -

256 ISPIGCLPAHLLGDMWGRFWTNLYSLTVPFGQKPNIDVTDAMVDQAWDAQRIFKEAEKFFVSVGLPNMTQGFWENSMLTDPGNVQ 340

341 KAVCHPTAWDLGKGDFRILMCTKVTMDDFLTAHHEMGHIOYDMAYAAQPELLRNGANEGFHEAVGEIMSLSAATPKHLKSIGLLS 425 1 -----MREAGWDKGGRILMCTKVTMDDFLTAHHEMGHIQYDMAYAAOPFLLRNGANEGFHEAVGEIMSLSAATPKHLKSIGLLS 79

426 PDFQEDNETEINELLKQALTIVGTLPFTYMLEKWRWMVFKGEIPKDQWMKKWWEMKREIVGVVEPVPHDETYCDPASLFHVSNDY 510

80 PDFQEDNETEINFLLKQALTIVGTLPFTYMLEKWRWMVFKGEIPKDQWMKKWWEMKREIVGVVEPVPHDETYCDPASLFHVSNDY 164

511 SEIRYYTRTLYQFQFQEALCQAAKHEGPLHKCDISNSTEAGQKLFNMLRLGKSEPWTLALENVVGAKNMNVRPLLNYFEPLFTWL 595 165 SEIRYYTRTLYQFQFQEALCQAAKHEGPLHKCDISNSTEAGQKLFNMLRLGKSEPWTLALENVVGAKNMNVRPLLNYFEPLFTWL 249

596 KDQNKNSFVGWSTDWSPYADQSIKVRISLKSALGDKAYEWNDNEMYLFRSSVAYAMRQYFLKVKNQMILFGEEDVRVANLKPRIS 680 250 KDQNKNSFVGWSTDWSPYADQSIKVRISLKSALGDKAYEWNDNEMYLFRSSVAYAMRQYFLKVKNQMILFGEEDVRVANLKPRIS 334

681 FNFFVTAPKNVSDIIPRTEVEKAIRMSRSRINDAFRLNDNSLEFLGIQPTLGPPNQPPVSIWLIVEGVVMGVIVVGIVILIETGI 765 335 FNEFVTAPKNVSDIIPRTEVEKAIRMSRSRINDAFRLNDNSLEFLGIQPTLGPPNQPPVSIWLIVEGVVMGVIVVGIVILIFTGI 419

766 RDRKKKNKARSGENPYASIDISKGENNPGEQNTDDVQTSF

420 RDRKKKNKARSGENPYASIDISKGENNPGEQNTDDVQTSF
805

459

Extended Data Fig. 6 | Protein sequence alignment of ACE2 and MIRb-ACE2. The predicted MIRb-ACE2 translation product is a 459 -amino acid protein lacking the indicted single peptide, domains interacting with SARS-CoV spike glycoprotein, but retaining the transmembrane domain. The novel 10-amino acid sequence created by LTR16A1 exonisation is also shown. 


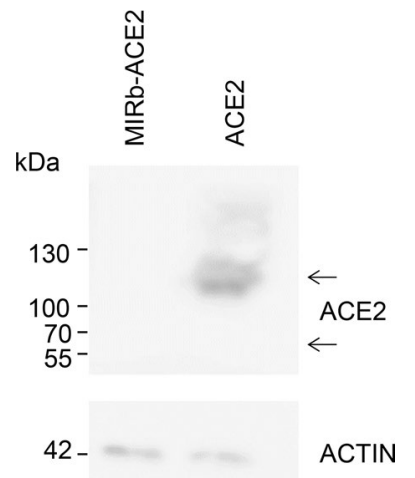

Extended Data Fig. 7 | Protein production by the ACE2 and MIRb-ACE2 transcript isoforms. Detection of ACE2 and putative MIRb-ACE2 protein product by Western blotting in cell lysates from HEK293T cells transfected to express either isoform. Proteins were detected using anti-ACE2 rabbit polyclonal antibodies (ab15348) as the primary reagent. One representative of 4 experiments is shown. 
ACE2

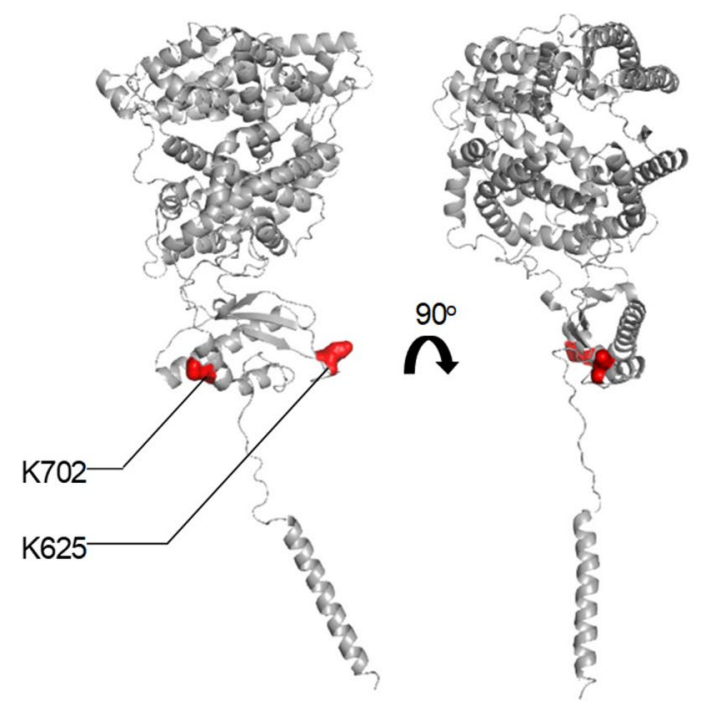

MIRb-ACE2

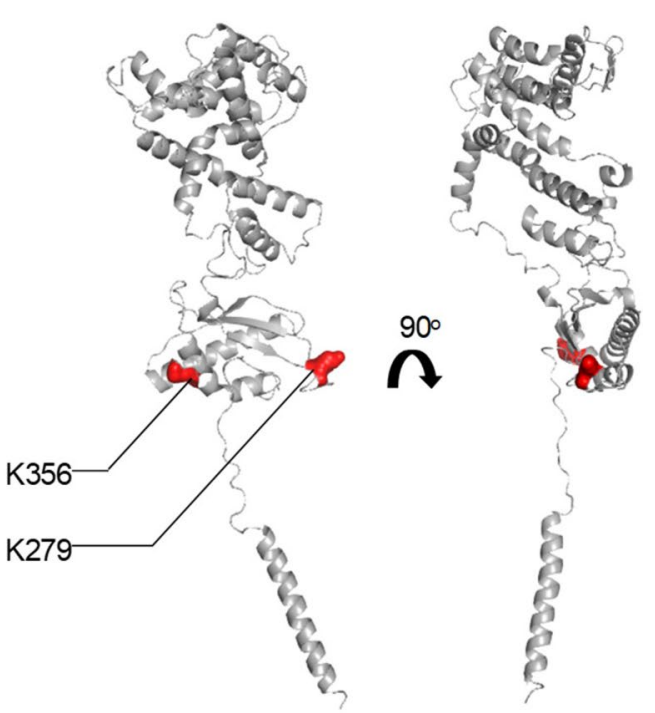

Extended Data Fig. 8 | Position of the ubiquitin targets in ACE2 and MIRb-ACE2 proteins. Structure of ACE2 (left) and predicted structure of the MIRb-ACE2 protein product (right) depicting the position of the two mutated $\mathrm{K}$ residues, targeted for ubiquitination. 
ACE2

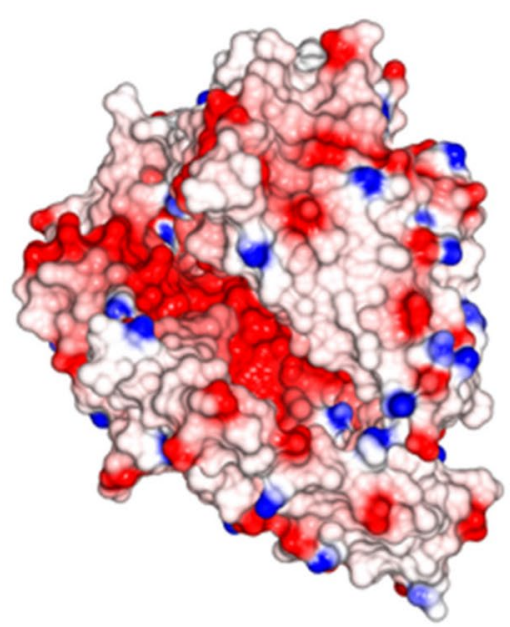

MIRb-ACE2

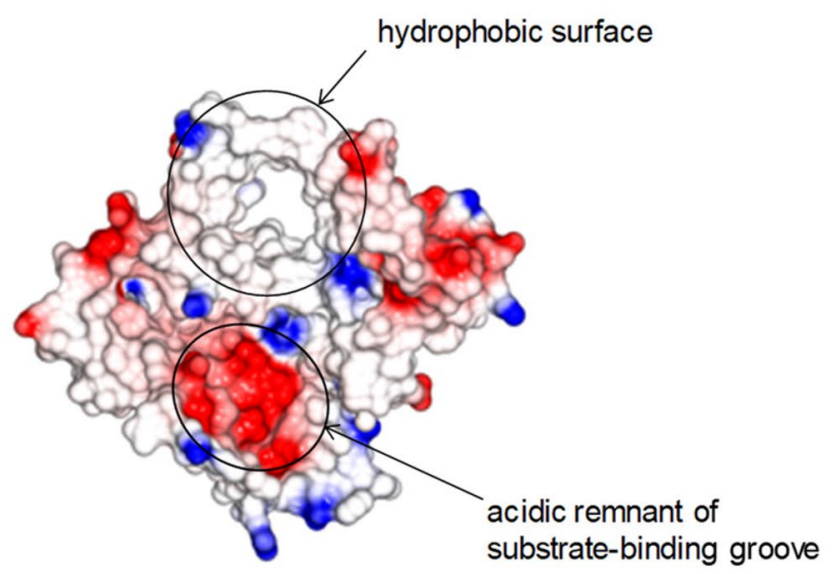

Extended Data Fig. 9 | Electrostatic-surface views of ACE2 and MIRb-ACE2 proteins. Matching projections of the canonical ACE2 (PDB ID: 6MOJ) and of the predicted MIRb-ACE2 protein product structures, if the latter were to exist in the same structural arrangement, but truncated. Basic, positively-charged residues shown in blue and acidic, negatively-charged residues in red. Arrows indicate an exposed hydrophobic surface and the open, acidic remnant of the substrate-binding groove. 


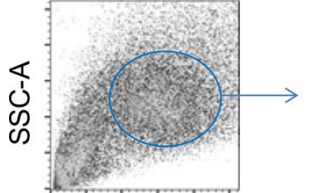

FSC-A

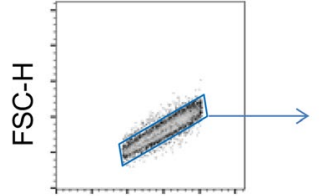

FSC-A

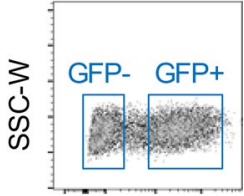

GFP

Extended Data Fig. 10 | Identification of cells expressing ACE2 or MIRb-ACE2 based on GFP reporter expression. Gating strategy for the identification of HEK293T cells transfected with plasmids encoding ACE2 or MIRb-ACE2 in conjunction with a P2A-GFP reporter. Expressing and non-expressing single cells were gated according to GFP expression. 


\section{Reporting Summary}

Nature Research wishes to improve the reproducibility of the work that we publish. This form provides structure for consistency and transparency in reporting. For further information on Nature Research policies, see our Editorial Policies and the Editorial Policy Checklist.

\section{Statistics}

For all statistical analyses, confirm that the following items are present in the figure legend, table legend, main text, or Methods section.

$\mathrm{n} / \mathrm{a}$ Confirmed

$\bigotimes$ The exact sample size $(n)$ for each experimental group/condition, given as a discrete number and unit of measurement

$\triangle$ A statement on whether measurements were taken from distinct samples or whether the same sample was measured repeatedly

The statistical test(s) used AND whether they are one- or two-sided

Only common tests should be described solely by name; describe more complex techniques in the Methods section.

Х $\square$ A description of all covariates tested

$\square$ A description of any assumptions or corrections, such as tests of normality and adjustment for multiple comparisons

$\square$ A full description of the statistical parameters including central tendency (e.g. means) or other basic estimates (e.g. regression coefficient)

AND variation (e.g. standard deviation) or associated estimates of uncertainty (e.g. confidence intervals)

$\square$ For null hypothesis testing, the test statistic (e.g. $F, t, r$ ) with confidence intervals, effect sizes, degrees of freedom and $P$ value noted

Give $P$ values as exact values whenever suitable.

Х $\square$ For Bayesian analysis, information on the choice of priors and Markov chain Monte Carlo settings

Х $\square$ For hierarchical and complex designs, identification of the appropriate level for tests and full reporting of outcomes

Х $\square$ Estimates of effect sizes (e.g. Cohen's d, Pearson's $r$ ), indicating how they were calculated

Our web collection on statistics for biologists contains articles on many of the points above.

\section{Software and code}

Policy information about availability of computer code

Data collection Western blots were visualized by chemiluminescence on an Amersham Imager 600 (GE Healthcare). Flow cytometry samples were run on a LSR Fortessa (BD Biosciences) running BD FACSDiva v8.0.

Data analysis Statistical comparisons were made using GraphPad Prism 7 (GraphPad Software) or SigmaPlot 14.0. RNAseq data from TCGA, GTEx, CCLE, and other studies were aligned to the cancer-tissue transcriptome assembly using GNU v3 parallel and Salmon v0.12.0. Splice junctions were visualised using the Integrative Genome Viewer v2.4.19. RNAseq reads were adapter trimmed and filtered for minimal $35 \mathrm{nt}$ sequences using Trimmomatic v0.39. Single cell RNA-seq reads were mapped with HISAT2. We used the DropSeq:picard toolbox (v2.3.0) to recapitulate processing of $\mathrm{HCL}$ samples as documented on 'https://github.com/ggjlab/HCL'. Amplicon sequencing reads were quality and adapter trimmed in pairs using cutadapt v1.18 and aligned with STAR v2.7.1a. Phylogenetic alignments were conducted with the MUSCLE aligner. Flow cytemetry data were analyzed with FlowJo v10 (Tree Star Inc.). All code used in this study has been previously described and cited within the Methods and is also available by request to the authors (G.K.).

For manuscripts utilizing custom algorithms or software that are central to the research but not yet described in published literature, software must be made available to editors and

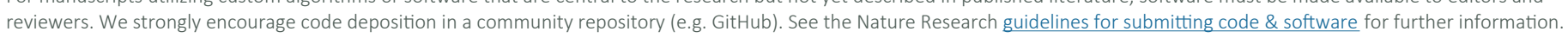


Policy information about availability of data

All manuscripts must include a data availability statement. This statement should provide the following information, where applicable:

- Accession codes, unique identifiers, or web links for publicly available datasets

- A list of figures that have associated raw data

- A description of any restrictions on data availability

Data supporting the findings of this study are available within the article and its supplementary information files and from the corresponding author upon reasonable request. Publicly available data were downloaded from the following databases: The Cancer Genome Atlas (TCGA) Research Network (http:// cancergenome.nih.gov), The Genotype-Tissue Expression (GTEx) Project (https://gtexportal.org/home); and the Broad Institute Cancer Cell Line Encyclopedia (CCLE) consortium (https://portals.broadinstitute.org/ccle). Additionally, RNA-seq data from individual studies (GSE147507 and GSE134355) were downloaded from the Gene Expression Omnibus (GEO) database (https://www.ncbi.nlm.nih.gov/geo). Source data are provided with this paper.

\section{Field-specific reporting}

Please select the one below that is the best fit for your research. If you are not sure, read the appropriate sections before making your selection. $\bigotimes$ Life sciences $\quad \square$ Behavioural \& social sciences $\quad \square$ Ecological, evolutionary \& environmental sciences

For a reference copy of the document with all sections, see nature.com/documents/nr-reporting-summary-flat.pdf

\section{Life sciences study design}

All studies must disclose on these points even when the disclosure is negative.

Sample size Sample size was determined empirically, based on the variability of the assays in our previous work. For example, the variability of NHBE cells to interferon treatment was based on prior results published in Major et al., (PMID: 32527928) and the transcriptional variability (determined by RT-qPCR) of CV-1, R9ab and MCA-38 cell cultures was based on prior results published in Ng et al., (PMID: 31729316).

Data exclusions No data were excluded.

\begin{tabular}{l|l} 
Replication & To ensure reproducibility, all experiments included 2-3 technical replicates within each independent experiment and most experiments
\end{tabular} (indicated in the article) were repeated at least twice (independent biological replicates). All attempts at replication for all experiments were successful.

Randomization Not applicable - the biological samples tested here were homogeneous cell lines and no pre-experiment differences required random allocation.

Blinding

Not applied - the behavior of the biological samples tested here (cell lines) is unaffected by the an unblinded design.

\section{Reporting for specific materials, systems and methods}

We require information from authors about some types of materials, experimental systems and methods used in many studies. Here, indicate whether each material, system or method listed is relevant to your study. If you are not sure if a list item applies to your research, read the appropriate section before selecting a response.

\begin{tabular}{|c|c|c|c|}
\hline \multicolumn{2}{|r|}{ Materials \& experimental systems } & \multicolumn{2}{|c|}{ Methods } \\
\hline $\mathrm{n} / \mathrm{a}$ & Involved in the study & $\mathrm{n} / \mathrm{a}$ & Involved in the study \\
\hline 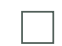 & $\bigotimes$ Antibodies & Х & $\square$ ChIP-seq \\
\hline [ & $\bigotimes$ Eukaryotic cell lines & $\square$ & \ Flow cytometry \\
\hline Х & $\square$ Palaeontology and archaeology & Х & $\square$ MRI-based neuroimaging \\
\hline$凶$ & $\square$ Animals and other organisms & & \\
\hline$凶$ & $\square$ Human research participants & & \\
\hline$\bigotimes$ & $\square$ Clinical data & & \\
\hline 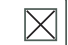 & $\square$ Dual use research of concern & & \\
\hline
\end{tabular}

\section{Antibodies}

Antibodies used

The following primary antibodies were used: anti-human ACE2 (ab15348, Abcam), anti-FLAG (F1804-50UG, clone M2, Sigma-Aldrich), HRP-conjugated anti-mouse IgG (\#7076, Cell Signaling Technology) or anti-rabbit IgG (\#7074, Cell Signaling Technology) and HRPconjugated anti-human actin (ab49900, Abcam).

Validation Antibodies were validated by the manufacturers. The anti-ACE2 rabbit polyclonal antibodies were validated by the manufacturer 


\section{Eukaryotic cell lines}

Policy information about cell lines

Cell line source(s)

HHEK293T (ATCC), A549 (ATCC), SCC-4 (ATCC), SCC-25 (ATCC), Vero (ATCC), CV-1(ATCC), MDCK (originally provided by Dr John McCauley, The Francis Crick Institute), R9ab (ATCC) and MCA-38 cells (originally provided by Dr. Giorgio Trinchieri, NCl, Bethesda, MD) were obtained from and verified as mycoplasma free by the Cell Services facility at the Francis Crick Institute. Primary human bronchial epithelial cells were purchased from Lonza.

Authentication

Established cell lines were validated by DNA fingerprinting by the Cell Services facility at the Francis Crick Institute

Mycoplasma contamination

Established cell lines were verified as mycoplasma-free by the Cell Services facility at the Francis Crick Institute

Commonly misidentified lines

(See ICLAC register)

No commonly misidentified cell lines were used in the study.

\section{Flow Cytometry}

Plots

Confirm that:

Х The axis labels state the marker and fluorochrome used (e.g. CD4-FITC).

Х The axis scales are clearly visible. Include numbers along axes only for bottom left plot of group (a 'group' is an analysis of identical markers).

Х All plots are contour plots with outliers or pseudocolor plots.

Х n numerical value for number of cells or percentage (with statistics) is provided.

\section{Methodology}

Sample preparation

Instrument

Software

Cell population abundance

Gating strategy
HEK293T cells were trypsinized into a single cell suspension and used directly for flow cytometry.

Samples were run on a LSR Fortessa (BD Biosciences).

Data were collected using FACSDiva v8.0 (BD Biosciences) and analyzed with FlowJo v10 (Tree Star Inc.).

HEK293T cells are a homogeneous cell line.

HEK293T cells are a homogeneous cell line and only the proportion of cells expressing GFP was enumerated. No additional gating was used.

$\bigotimes$ Tick this box to confirm that a figure exemplifying the gating strategy is provided in the Supplementary Information. 